\title{
Exponential parameterization of wave functions for quantum dynamics: Time- dependent Hartree in second quantization
}

Niels Kristian Madsen, Mads Bøttger Hansen, Alberto Zoccante, Kasper Monrad, Mikkel Bo Hansen, and Ove Christiansen

Citation: J. Chem. Phys. 149, 134110 (2018); doi: 10.1063/1.5049344

View online: https://doi.org/10.1063/1.5049344

View Table of Contents: http://aip.scitation.org/toc/jcp/149/13

Published by the American Institute of Physics

\section{Articles you may be interested in}

Communication: Can excitation energies be obtained from orbital energies in a correlated orbital theory?

The Journal of Chemical Physics 149, 131101 (2018); 10.1063/1.5052442

Semiclassical transition state theory based on fourth order vibrational perturbation theory: Model system studies beyond symmetric Eckart barrier

The Journal of Chemical Physics 149, 134109 (2018); 10.1063/1.5040978

Perspective: Computational chemistry software and its advancement as illustrated through three grand challenge cases for molecular science

The Journal of Chemical Physics 149, 180901 (2018); 10.1063/1.5052551

Accurate double excitations from ensemble density functional calculations

The Journal of Chemical Physics 149, 134103 (2018); 10.1063/1.5043411

Accurate quantum molecular dynamics for multidimensional systems by the basis expansion leaping multiconfiguration Gaussian (BEL MCG) method

The Journal of Chemical Physics 149, 134113 (2018); 10.1063/1.5046643

Communication: The pole structure of the dynamical polarizability tensor in equation-of-motion coupled-

cluster theory

The Journal of Chemical Physics 149, 141101 (2018); 10.1063/1.5053727

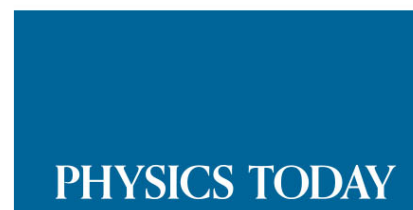

WHITEPAPERS
ADVANCED LIGHT CURE ADHESIVES

Take a closer look at what these environmentally friendly adhesive systems can do

\section{READ NOW}

PRESENTED BY

(8) MASTERBOND' 


\title{
Exponential parameterization of wave functions for quantum dynamics: Time-dependent Hartree in second quantization
}

\author{
Niels Kristian Madsen, Mads Bøttger Hansen, Alberto Zoccante, ,a) Kasper Monrad, \\ Mikkel Bo Hansen, and Ove Christiansen ${ }^{\text {b) }}$ \\ Department of Chemistry, University of Aarhus, Langelandsgade 140, DK-8000 Aarhus C, Denmark
}

(Received 20 July 2018; accepted 17 September 2018; published online 4 October 2018)

\begin{abstract}
We derive equations for describing the time evolution of variational wave functions in linear and exponential parameterization with a second-quantization (SQ) formulation. The SQ formalism covers time-dependent Hartree (TDH), while exact states and approximate vibrational configuration interaction wave functions are described using state-transfer operators. We present detailed expressions for efficient evaluation of TDH in linear (L-TDH) and exponential (X-TDH) parametrization and an efficient implementation supporting linear scaling with respect to the number of degrees of freedom $M$ when the Hamiltonian operator contains a constant number of terms per mode independently of the size of the system. The computational cost of the X-TDH method is reduced significantly compared to the L-TDH method for systems with many operator terms per mode such as is typical for accurate molecular potential-energy surfaces. Numerical results for L-TDH and X-TDH are presented which confirm the theoretical reduction of the $M$ scaling compared to standard first-quantization formulations. Calculations on Henon-Heiles potentials with more than $10^{5}$ dimensions and polycyclic aromatic hydrocarbons with up to 264 modes have been performed. Thus, the SQ formulation and the X-TDH method pave the way for studying the time-resolved quantum dynamics of large molecules. Published by AIP Publishing. https://doi.org/10.1063/1.5049344
\end{abstract}

\section{INTRODUCTION}

Many interesting phenomena in chemical physics are best described using a time-dependent quantum-mechanical formulation. ${ }^{1}$ The numerical calculation of accurate approximate time-dependent wave functions is therefore an important theoretical challenge. For few-dimensional problems, there exist standard and sufficiently efficient approaches. ${ }^{1,2}$ However, for only slightly more degrees of freedom (more than four atoms), matters are complicated, and over the years, many methods have been developed employing different ideas for how to parameterize wave functions and how to propagate these in time.

One of the basic methods for computing the time evolution of a molecular system is the time-dependent Hartree (TDH) approach, also sometimes denoted as the time-dependent selfconsistent-field (TD-SCF). TDH is the time-dependent analog of vibrational self-consistent-field (VSCF), ${ }^{3-7}$ which in turn is the analog of Hartree-Fock theory, only now for distinguishable degrees of freedom corresponding to nuclear motion. The fundamental approximation of TDH is the adoption of a single Hartree product as a wave-function ansatz which leads to a mean-field description of the interactions between the different degrees of freedom. Being a mean-field approximation, TDH has significant shortcomings. ${ }^{2,8}$ Nevertheless, TDH and many of its theoretical and technical ingredients are essential

\footnotetext{
a)Present address: Dipartimento di Scienze e Innovazione Tecnologica, DiSIT, University del Piemonte Orientale “Amedeo Avogadro," Alessandria, Italy. b)Electronic mail: ove@chem.au.dk
}

parts of building up general and efficient quantum-dynamical machinery.

Among theoretical methods proceeding beyond TDH, we mention in particular multi-configuration time-dependent Hartree (MCTDH), ${ }^{8,9}$ which is now a well-established and versatile theory and computational method for calculating time-dependent wave functions. MCTDH combines the timedependent variational principle (TDVP) with evolution of (now several) Hartree products and thus shares a number of basic ideas and practical components with TDH, such as mean-field matrices. Although MCTDH does not overcome the exponential growth of computational cost with increasing dimensionality, the method considerably alleviates the issue by a variational optimization of the one-mode basis functions in a manner that can be systematically improved to the numerically exact limit. Still, the inherent exponential scaling with the number of degrees of freedom is a potential limitation, and several suggestions have been put forward to mitigate this, such as multilayer $\mathrm{MCTDH},{ }^{10-12}$ or alternative approaches tailored to specific types of complex systems such as system-bath simulations. ${ }^{13,14}$ Multi-configurational expansions have been combined with a time-dependent Gaussian basis or similar in various forms, such as the G-MCTDH ${ }^{15}$ and variational multi-configurational Gaussian (vMCG) (see Ref. 16 for a review), and (two-layer) coupled-coherent states (CCS). ${ }^{17}$ These methods are in turn somewhat related to other successful Gaussian-based approaches such as the multiple spawning methods; see Ref. 18 for a very recent review. A fundamental distinction is whether the basis has its own time evolution (i.e., classical Gaussians) or is described in terms 
of parameters determined by (variational) criteria applied to the full wave functions. Altogether there is still room for new ways of addressing the constituting elements of computational quantum dynamics.

For time-independent wave functions, we have developed a vibrational coupled-cluster (VCC) theory. ${ }^{19,20}$ The VCC method has proven to be an efficient method for calculating anharmonic vibrational spectra and properties of molecules, ${ }^{21,22}$ and there has been significant progress toward higher efficiency and larger molecular systems, e.g., by employing tensor decomposition methods. ${ }^{23}$ As we currently seek to generalize this approach to a time-dependent framework, it is important to be able to describe the reference state for the cluster expansion (a Hartree-product state like VSCF/TDH) as well as general time-dependent transformations of the basis.

In this paper, we explore new ways of parameterizing time-dependent wave functions and formulating equations for the time evolution of quantum states. Specifically, we investigate the consequences of adopting an exponential parameterization and second quantization (SQ) in conjunction with the TDVP. Our formulation covers TDH, exact wave functions, and time-dependent vibrational configuration interaction (VCI). TDH is studied in significant detail for several reasons, including (i) the general theoretical significance due to its status as a fundamental approximation in chemical physics, (ii) its direct or indirect role in related theoretical frameworks such as those mentioned above, and (iii) the use of TDH methodology as a vehicle for pushing the size-limits for quantum simulations.

In this work, we employ the previously introduced secondquantization (SQ) formulation of many-mode dynamics. ${ }^{7}$ The SQ formulation requires, for a start, a significant amount of initial considerations and is in no way intended as a short cut for TDH compared to existing first-quantization (FQ) formulations (for FQ derivations of TDH, see Refs. 24 and 25). However, once established, the SQ formulation provides a convenient framework supporting and inspiring alternative formulations, implementations, and extensions. The SQ formulation has in this regard previously proved useful, for example, by automatically reducing the computational complexity by one order in VSCF theory ${ }^{6}$ which is specifically interesting due to the close relation between VSCF and TDH. We use the SQ formulation to provide useful general relations for deriving time-dependent equations and carrying out the time evolution of operators and one-mode functions. We use these to formulate operator-based equations for describing the time evolution of the TDH state in terms of equations of motion (EOMs) for the SQ creation operators. With a linear parameterization of the time evolution of the SQ creation operators (or correspondingly the one-mode functions), these lead to the linear TDH (L-TDH) equations requiring the full mean-field matrix for their integration. We subsequently present a derivation and analysis of the EOMs for an exponentially parameterized TDH state, an approach we denote X-TDH, completing the initial considerations of Ref. 26. Exponential parameterizations have previously been highly useful in electronic structure theory for non-redundant transformations of orbitals or other quantities. ${ }^{27}$ Expressing the unitary time evolution in terms of the elements of an anti-Hermitian matrix by ansatz preserves orthogonality of the basis functions. A mathematical analysis of the parameterization reveals how the actual transformations can efficiently be carried out and identifies periodicities and thereby the relevant parameter ranges. The time-derivative potentially results in quite involved equations for an exponential parameterization, but from a mathematical analysis, it becomes clear that it is natural to consider the parameter space in terms of projections on some specific directions. Employing these projections, very simple final equations are obtained for the time evolution of the X-TDH parameters. Similar to L-TDH, the X-TDH EOMs can be integrated without explicitly solving linear equations and/or inverting matrices which is a non-trivial result. In addition, $\mathrm{X}$-TDH does not require the full mean-field matrix like L-TDH, offering significant computational savings.

TDH has the potential of being applicable to large systems due to its low computational scaling, but realizing this potential for real molecules is a significant challenge. We have previously illustrated how VSCF can be applied to systems with thousands of degrees of freedom, ${ }^{6}$ and one of the goals of the present formulation is to be able to carry over these technical advances to time-dependent wave functions. We illustrate how the active-term algorithm of Ref. 6 can be employed to provide highly efficient implementations of both L-TDH and $\mathrm{X}-\mathrm{TDH}$. A low and predictable computational scaling with the size of the system for Hamiltonians with a restricted level of mode-coupling is obtained for both L-TDH and X-TDH and illustrated in practice by applying the implementation to polycyclic aromatic hydrocarbons (PAHs) with up to 264 modes. The efficiency gain of X-TDH compared to L-TDH is clearly seen for operators with many terms. Linear scaling is achieved for both X-TDH and L-TDH in the limit where the number of non-zero terms per mode is constant, making computations with more than $10^{5}$ modes possible.

The paper is structured as follows. Section II describes the theoretical developments with Secs. II A and II B introducing the TDVP and the SQ formulation, respectively. In Sec. III, a general TDH EOM is derived, which covers both linear and exponential parametrizations, and explicit EOMs for the L-TDH method are presented. In Sec. IV, EOMs for a general exponential parametrization are derived and concrete expressions for X-TDH are presented. Section V presents EOMs for an exponential parametrization of the exact method as well as approximate time-dependent VCI. Section VI describes the implementation, while our numerical results are presented in Sec. VII. Finally, Sec. VIII provides a summary and future outlook.

\section{GENERAL THEORY}

A time-dependent wave function can be written in a phaseseparated form as

$$
\bar{\Psi}\left(q_{1}, \ldots, q_{M}, t\right)=\exp (-i F(t)) \tilde{\Psi}\left(q_{1}, \ldots, q_{M}, t\right),
$$

where we consider a system with $M$ degrees of freedom (modes) with coordinates $\left\{q_{1}, q_{2}, \ldots, q_{M}\right\}$. The phase-isolated wave function, $\tilde{\Psi}$, can be expressed as a linear combination 
of Hartree products, $\tilde{\Phi}_{\mathbf{r}}$, of one-mode functions (modals), $\left\{\tilde{\phi}_{r^{m}}^{m}\left(q_{m}, t\right)\right\}$,

$$
\tilde{\Psi}\left(q_{1}, \ldots, q_{M}, t\right)=\sum_{\mathbf{r}} C_{\mathbf{r}}(t) \tilde{\Phi}_{\mathbf{r}}\left(q_{1}, \ldots, q_{M}, t\right)
$$

with

$$
\tilde{\Phi}_{\mathbf{r}}\left(q_{1}, \ldots, q_{M}, t\right)=\prod_{m=1}^{M} \tilde{\phi}_{r^{m}}^{m}\left(q_{m}, t\right) .
$$

In the TDH approximation, a single Hartree product is used as a wave-function ansatz. The time dependence of the modals is not strictly necessary for multi-configurational wave functions, but it is introduced in, e.g., MCTDH in order to obtain a more compact form of the wave function.

\section{A. The time-dependent variational principle}

A variational solution to the time-dependent Schrödinger equation (TDSE) is obtained by applying the TDVP to the phase-separated wave function of (1). This results in EOMs for the generalized phase factor $F(t)$ and the phase-isolated wave function. One formulation of the TDVP is the least-action variational principle, ${ }^{28-31}$

$$
\delta S \equiv \delta \int_{t_{0}}^{t_{1}} \mathcal{L} d t=0 \quad \text { with } \quad \mathcal{L} \equiv\left\langle\bar{\Psi}\left|\left(H-i \frac{\partial}{\partial t}\right)\right| \bar{\Psi}\right\rangle,
$$

where $\delta \bar{\Psi}\left(t_{0}\right)=\delta \bar{\Psi}\left(t_{1}\right)=0$. For a phase-separated wave function as in Eq. (1), this results in the equations

$$
\begin{aligned}
& \dot{F}=\left\langle\tilde{\Psi}\left|\left(H-i \frac{\partial}{\partial t}\right)\right| \tilde{\Psi}\right\rangle, \\
& 0=\operatorname{Re}\left\langle\delta \tilde{\Psi}\left|\left(H-i \frac{\partial}{\partial t}\right)\right| \tilde{\Psi}\right\rangle,
\end{aligned}
$$

where we have required normalization at all times, i.e., $\delta\langle\tilde{\Psi} \mid \tilde{\Psi}\rangle=2 \operatorname{Re}\langle\delta \tilde{\Psi} \mid \tilde{\Psi}\rangle=0$. The above equations can equally well be derived from projecting the Schrödinger equation on allowed variations and requiring that if $\delta \tilde{\Psi}$ is an allowed variation so is $i \delta \tilde{\Psi}$. This results in the same equation for $\dot{F}$ and the alternative (but equivalent) expression for determining the time evolution of the wave-function parameters, ${ }^{32,33}$

$$
0=\left\langle\delta^{\perp} \tilde{\Psi}\left|\left(H-i \frac{\partial}{\partial t}\right)\right| \tilde{\Psi}\right\rangle,
$$

using orthogonal variations $\left\langle\delta^{\perp} \tilde{\Psi} \mid \tilde{\Psi}\right\rangle=0$.

\section{B. Second-quantization formulation of many-mode theory}

In the second-quantization (SQ) formulation of Ref. 7, vibrational wave functions and operators are described in terms of creation and annihilation operators. The one-mode creation operator, $a_{p^{m}}^{m \dagger}$, creates occupation in the modal labeled with $p^{m}$ for mode $m$. Likewise, the Hermitian-conjugate operator, the annihilation operator, removes the modal. The operators satisfy the commutator relations,

$$
\begin{aligned}
{\left[a_{r^{m}}^{m}, a_{s^{m^{\prime}}}^{m^{\prime} \dagger}\right.} & =\delta_{m m^{\prime}} \delta_{r^{m} s^{m^{\prime}}}, \\
{\left[a_{r^{m}}^{m}, a_{s^{m^{\prime}}}^{m^{\prime}}\right.} & =0, \\
{\left[a_{r^{m}}^{m \dagger}, a_{s^{m^{\prime}}}^{m^{\prime} \dagger}\right] } & =0 .
\end{aligned}
$$

The state with no occupation is denoted as the vacuum state, $|v a c\rangle$, which satisfies $\langle$ vaclvac $\rangle=1$ and the killer condition,

$$
a_{r^{m}}^{m}|\mathrm{vac}\rangle=0 \text {. }
$$

We emphasize that this set of operators is fundamentally different from the ladder operators used in the harmonicoscillator problem, and the formulation is in no way restricted to harmonic-oscillator basis functions or reference states. Hartree-product states are in second quantization represented by a string of one-mode creation operators applied to the vacuum state. Thus the TDH state can be written as

$$
\left|\tilde{\Phi}_{\mathbf{i}}\right\rangle=\prod_{m=1}^{M} \tilde{a}_{i^{m}}^{m \dagger}|\mathrm{vac}\rangle .
$$

Here the $M$-dimensional index vector $\mathbf{i}=\left(i_{1}, i_{2}, \ldots, i_{M}\right)$ defines which particular modal is occupied for each mode. Note that we allow ourselves to use the same state labels in first and second quantization though two different formal representations are actually in play. In the following, we will omit the $\mathbf{i}$ subscript from the TDH wave function.

The following convention is adopted in the labelling of modals: $i^{m}$ is the reference modal for the $m$ th mode; $a^{m}, b^{m}$, $c^{m}, d^{m}$ denote the virtual modals; and $p^{m}, q^{m}, r^{m}, s^{m}$ denote modals of unspecified occupancy.

\section{A note on commutator relations}

We may consider Eqs. (7)-(9) to be canonical commutator relations for creation and annihilation operators, or we could say that the creation and annihilation operators are in some sense canonical since they are directly tied to the underlying modals. We will, however, encounter other creation and annihilation operators, such as similarity-transformed ones or time derivatives of ones; in either case, we have the following important result.

If any annihilation operator $\tilde{a}_{r^{m}}^{m}$ and creation operator $\tilde{a}_{s^{m}}^{m \dagger}$ are ultimately a linear combination of the canonical annihilation and creation operators of (7), i.e.,

$$
\begin{aligned}
\tilde{a}_{r^{m}}^{m} & =\sum_{p^{m}} c_{p^{m}} a_{p^{m}}^{m}, \\
\tilde{a}_{s^{m}}^{m \dagger} & =\sum_{q^{m}} d_{q^{m}} a_{q^{m}}^{m \dagger},
\end{aligned}
$$

then their commutator is a scalar operator (i.e., a scalar times the identity operator) because

$$
\left[\tilde{a}_{r^{m}}^{m}, \tilde{a}_{s^{m}}^{m \dagger}\right]=\sum_{p^{m} q^{m}} c_{p^{m}} d_{q^{m}}\left[a_{p^{m}}^{m}, a_{q^{m}}^{m \dagger}\right]
$$

which is a sum of scalars.

\section{Operators in second quantization}

The creation and annihilation operators are used to express all states as well as all other operators. For future reference, we introduce the one-mode shift operator,

$$
E_{r^{m} s^{m}}^{m}=a_{r^{m}}^{m \dagger} a_{s^{m}}^{m},
$$

which shifts occupation from level $s^{m}$ to $r^{m}$ in mode $m$. The shift operators satisfy the commutator relation, 


$$
\left[E_{p^{m} q^{m}}^{m}, E_{r^{\prime m^{\prime}} s^{\prime m^{\prime}}}^{m^{\prime}}\right]=\delta_{m m^{\prime}}\left(\delta_{q^{m} r^{\prime m}} E_{p^{m} s^{m}}^{m}-\delta_{p^{m} s^{\prime m}} E_{r^{\prime m} q^{m}}^{m}\right)
$$

A general Hermitian operator of the form $\Omega=\Omega_{0}+\sum_{m} \Omega^{m}$ $+\cdots$ can in SQ be expressed as

$$
\begin{aligned}
& \Omega=\Omega_{0}+\sum_{m} \sum_{r^{m} s^{m}} \Omega_{r^{m} s^{m}}^{m} E_{r^{m} s^{m}}^{m}+\sum_{m_{1}<m_{2}} \sum_{r^{m_{1}}} \sum_{r^{m_{2}} s^{m_{1}} s^{m_{2}}}
\end{aligned}
$$

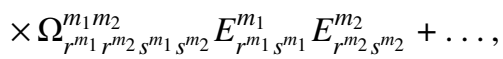

with integrals

$$
\Omega_{r^{m_{1}} r^{m_{2}} \ldots m^{m_{n}} s^{m_{1}} s^{m_{2}} \ldots s^{m_{n}}}^{m_{1} m_{2}}=\left\langle r^{m_{1}} r^{m_{2}} \ldots r^{m_{n}}\left|\Omega^{m_{1} m_{2} \ldots m_{n}}\right| s^{m_{1}} s^{m_{2}} \ldots s^{m_{n}}\right\rangle
$$

The exact vibrational Hamiltonian can be expressed by such an expansion. However, since the exact potential-energy surface (PES) of the system is not generally known, we often choose to represent the Hamiltonian in the computationally efficient sum-over-products (SOP) form

$$
H=\sum_{t} c_{t} \prod_{m \in \mathbf{m}_{t}} h^{m t},
$$

where $h^{m t}$ is a one-mode operator on mode $m$. The SOP form has been shown to be computationally convenient, e.g., in the cases of MCTDH, ${ }^{8}$ VCC, ${ }^{19,20,23}$ and VSCF. ${ }^{6}$ The one-mode operators can be expressed in either the time-independent or the time-dependent basis (indicated by a tilde) as

$$
h^{m t}=\sum_{r^{m} s^{m}} h_{r^{m} s^{m}}^{m t} a_{r^{m}}^{m \dagger} a_{s^{m}}^{m}=\sum_{r^{m} s^{m}} \tilde{h}_{r^{m} s^{m}}^{m t}(t) \tilde{a}_{r^{m}}^{m \dagger}(t) \tilde{a}_{s^{m}}^{m}(t) .
$$

Here the time evolution of integrals is such as to counteract the evolution of the creation/annihilation operators, thus at all times reproducing the same operator.

\section{Unitary transformations in second quantization}

Unitary transformations in the modal space can be described using the one-mode shift operators in analogy with orbital transformations in electronic structure theory. Operators of the form

$$
U(\kappa)=\exp (\kappa)=\exp \left(\sum_{m} \kappa^{m}\right)=\exp \left(\sum_{m} \sum_{r^{m} s^{m}} \kappa_{r^{m} s^{m}}^{m} E_{r^{m} s^{m}}^{m}\right)
$$

represent modal transformations (see Ref. 7 for a proof) and are unitary, provided the operators $\kappa^{m}$ and the corresponding matrix representations on the modal spaces $\mathbf{K}^{m}$ are anti-Hermitian. Unitary transformations can therefore be parametrized by the elements of the $\mathbf{K}^{m}$ matrices, where we note that each mode has its own transformation matrix and there are no $\kappa$ parameters referring to more than one mode at a time. Consider now the set of Hartree-products that can be described in a given orthonormal basis. Any Hartree-product state can be obtained by applying an exponential operator as the one given in Eq. (20) to an arbitrary Hartree-product state. In the case where there is only one mode, the TDH treatment is exact and thus covers exact states. Furthermore, a formulation equivalent to this one-mode case is also applicable-using state transfer operators-to the exact many-mode limit as well as the approximate VCI case, as is briefly discussed in Sec. V.

In Eq. (1), we separate out a total phase of the wave function. Correspondingly, we shall later limit ourselves to special unitary transformations of the phase-isolated wave function, which means that the $\mathbf{K}^{m}$ matrices are special unitary matrices that have zero diagonal elements and unit determinants. The diagonal elements describe complex phase shifts, but we use the $F$ factor in Eq. (1) for describing the overall phase of the total wave function, thereby circumventing redundant descriptions of phase, meaning we have one overall phase factor as opposed to a phase factor for each mode.

The unitary-transformation operators of Eq. (20) are used to rotate the creation and annihilation operators to a new basis,

$$
e^{\kappa^{m}} E_{r^{m} s^{m}}^{m} e^{-\kappa^{m}}=e^{\kappa^{m}} a_{r^{m}}^{m \dagger} e^{-\kappa^{m}} e^{\kappa^{m}} a_{s^{m}}^{m} e^{-\kappa^{m}}=\tilde{a}_{r^{m}}^{m \dagger} \tilde{a}_{s^{m}}^{m} .
$$

This defines the similarity-transformed operators,

$$
\begin{aligned}
& \tilde{a}_{r^{m}}^{m \dagger} \equiv e^{\kappa^{m}} a_{r^{m}}^{m \dagger} e^{-\kappa^{m}}=\sum_{p^{m}} a_{p^{m}}^{m \dagger}\left[e^{\mathbf{K}^{m}}\right]_{p^{m} r^{m}}, \\
& \tilde{a}_{s^{m}}^{m} \equiv e^{\kappa^{m}} a_{s^{m}}^{m} e^{-\kappa^{m}}=\sum_{p^{m}}\left[e^{-\mathbf{K}^{m}}\right]_{s^{m} p^{m}} a_{p^{m}}^{m},
\end{aligned}
$$

where in the case of $\mathrm{TDH}$, the $\mathbf{K}^{m}$ matrices contain the time-dependent wave-function parameters. The similaritytransformed creation and annihilation operators are used to represent operators and wave functions in the time-dependent basis. In order to fulfill Eq. (19), the transformation of the integrals of the Hamiltonian operator must be defined as

$$
\tilde{h}_{r^{m} s^{m}}^{m t}=\sum_{p^{m} q^{m}}\left[e^{-\mathbf{K}^{m}}\right]_{r^{m} p^{m}} h_{p^{m} q^{m}}^{m t}\left[e^{\mathbf{K}^{m}}\right]_{q^{m} s^{m}} .
$$

Since the $e^{\mathbf{K}^{m}}$ and $e^{-\mathbf{K}^{m}}$ matrices are unitary, they can alternatively be written as $\mathbf{U}^{m}$ and $\mathbf{U}^{m \dagger}$ and equations-of-motion can be derived for the $U^{m}$-matrix elements instead of the $\kappa_{r^{m} s^{m}}^{m}$ parameters. This approach is taken in Sec. III B, while the EOMs for the $\kappa_{r^{m} s^{m}}^{m}$ parameters are derived in Sec. IV.

\section{The resolution of the identity in second quantization}

In principle, the SQ formalism as introduced so far allows for arbitrary (non-negative integral) occupancies in the same mode, even in the same modal, although these have no firstquantization counterparts. We will, however, limit ourselves to physically relevant occupancies, i.e., states that are at most 
singly occupied in any mode (but which can still be unoccupied as well).

Under this assumption, it holds true that any two annihilation operators of the same mode applied to any physical state $|\alpha\rangle$ give zero since at least one of them will operate directly on the vacuum state as in (10),

$$
a_{r^{m}}^{m} a_{s^{m}}^{m}|\alpha\rangle=0 .
$$

The commutation relation (7) reflects the orthonormality of the 1-mode states $\left\{\left|r^{m}\right\rangle\right\}$ since

$$
\left\langle r^{m} \mid s^{m}\right\rangle=\left\langle\operatorname{vac}\left|a_{r^{m}}^{m} a_{s^{m}}^{m \dagger}\right| \operatorname{vac}\right\rangle=\left\langle\operatorname{vac}\left|\left[a_{r^{m}}^{m}, a_{s^{m}}^{m \dagger}\right]\right| \operatorname{vac}\right\rangle=\delta_{r^{m} s^{m}} .
$$

The identity operator can therefore be resolved as the orthogonal projection operator onto all possible physical states obtained as a direct product of these 1-mode states,

$$
\begin{aligned}
I= & |\mathrm{vac}\rangle\left\langle\mathrm{vac}\left|+\sum_{m_{0}} \sum_{r^{m_{0}}}\right| r^{m_{0}}\right\rangle\left\langle r^{m_{0}}\right| \\
& +\sum_{m_{0}<m_{1}} \sum_{r^{m_{0}, r^{m_{1}}}}\left|r^{m_{0}} r^{m_{1}}\right\rangle\left\langle r^{m_{0}} r^{m_{1}}\right|+\cdots \\
= & \sum_{\mathbf{m} \subset \mathbb{N}} \sum_{\mathbf{r}^{\mathbf{m}}}\left|\mathbf{r}^{\mathbf{m}}\right\rangle\left\langle\mathbf{r}^{\mathbf{m}}\right| \\
= & \sum_{\mathbf{m} \subset \mathbb{N}} \sum_{\mathbf{r}^{\mathbf{m}}}\left(\prod_{m \in \mathbf{m}} a_{r^{m}}^{m \dagger}\right)|\operatorname{vac}\rangle\langle\operatorname{vac}|\left(\prod_{m \in \mathbf{m}} a_{r^{m}}^{m}\right) .
\end{aligned}
$$

\section{Projection operators in second quantization}

In accord with the discussion of the resolution of the identity, we can introduce one-mode projection operators. By a one-mode projection operator $P_{q^{m}}^{m}$ for the $q^{m}$ 'th state of mode $m$, we understand a projection onto all states (no matter how many modes are occupied) that have unit occupancy for that state, i.e.,

$$
\begin{aligned}
P_{q^{m}}^{m} & =\left|q^{m}\right\rangle\left\langle q^{m}\left|+\sum_{m_{0} \neq m} \sum_{r^{m_{0}}}\right| r^{m_{0}} q^{m}\right\rangle\left\langle r^{m_{0}} q^{m}\right|+\cdots \\
& =\sum_{\mathbf{m} \subset\{\mathbb{N} \backslash m\}} \sum_{\mathbf{r}^{\mathbf{m}}}\left(\prod_{m^{\prime} \in \mathbf{m}} a_{r^{m^{\prime}}}^{m^{\prime} \dagger}\right) a_{q^{m}}^{m \dagger}|\operatorname{vac}\rangle\langle\operatorname{vac}| a_{q^{m}}^{m}\left(\prod_{m^{\prime} \in \mathbf{m}} a_{r^{m^{\prime}}}^{m^{\prime}}\right) .
\end{aligned}
$$

Now, under the assumption of (25), we without consequence can add to $P_{q^{m}}^{m}$ the sum over terms for subsets of $\mathbb{N}$ containing $m$,

$$
\sum_{\mathbf{m} \subset\{\mathbb{N} \backslash m\}} \sum_{\mathbf{r}^{\mathbf{m} \cup\{m\}}}\left(\prod_{m^{\prime} \in \mathbf{m} \cup\{m\}} a_{r^{m^{\prime}}}^{m^{\prime} \dagger}\right) a_{q^{m}}^{m \dagger}|\operatorname{vac}\rangle\langle\operatorname{vac}| a_{q^{m}}^{m}\left(\prod_{m^{\prime} \in \mathbf{m} \cup\{m\}} a_{r^{m^{\prime}}}^{m^{\prime}}\right),
$$

because all these terms will give zero when applied to any physically relevant state due to the double annihilation in mode $m$. Adding these terms to the expression for $P_{q^{m}}^{m}$ turns the sum over $\mathbf{m} \subset\{\mathbb{N} \backslash m\}$ into a sum over all subsets $\mathbf{m} \subset \mathbb{N}$, and pulling out the operators $a_{q^{m}}^{m \dagger}$ and $a_{q^{m}}^{m}$, common to all terms, we obtain an expression with the resolution of the identity and get

$$
\begin{aligned}
P_{q^{m}}^{m} & =a_{q^{m}}^{m \dagger} \underbrace{\left[\sum_{\mathbf{m} \subset \mathbb{N}} \sum_{\mathbf{r}^{\mathbf{m}}}\left(\prod_{m^{\prime} \in \mathbf{m}} a_{r^{m^{\prime}}}^{m^{\dagger} \dagger}|\operatorname{vac}\rangle\langle\operatorname{vac}|\left(\prod_{m^{\prime} \in \mathbf{m}} a_{r^{m^{\prime}}}^{m^{\prime}}\right)\right]\right.}_{=I} a_{q^{m}}^{m} \\
& =a_{q^{m}}^{m \dagger} a_{q^{m}}^{m} .
\end{aligned}
$$

Defining the projector $P^{m}=\sum_{q^{m}} P_{q^{m}}^{m}$ onto states that have occupancy in mode $m$ leads to the definition of the orthogonalprojection operator,

$$
O_{q^{m}}^{m}=P^{m}-P_{q^{m}}^{m}
$$

\section{SECOND-QUANTIZATION DERIVATION OF TDH}

$\mathrm{TDH}$ is based on a single-configuration wave-function ansatz,

$$
|\bar{\Phi}(t)\rangle=\exp (-i F(t))|\tilde{\Phi}(t)\rangle=\exp (-i F(t)) \prod_{m=1}^{M} \tilde{a}_{i^{m}}^{m^{\dagger}}(t)|\mathrm{vac}\rangle,
$$

where $\tilde{a}_{i^{m}}^{m^{\dagger}}(t)$ is a creation operator in the time-dependent basis.

Phase factors and normalization factors can be transferred between the modes and the $\exp (-i F)$ factor without changing the total wave functions. The imaginary part of $F$ ensures conservation of norm if it is determined by the time-dependent Schrödinger equation. We shall fix from the outset the normalization by requiring the time-dependent creation and annihilation operators to fulfill the standard commutator relations [Eqs. (7)-(9)] and thereby require $F$ to be real.

In order to maintain an orthonormal one-mode basis during the wave-packet propagation, we define Hermitian constraint operators $g^{m}=\sum_{r^{m} s^{m}} \tilde{g}_{r^{m} s^{m}}^{m} \tilde{a}_{r^{m}}^{m \dagger} \tilde{a}_{s^{m}}^{m}$ with the property,

$$
\left[\tilde{a}_{r^{m}}^{m}(t), \dot{\tilde{a}}_{s^{m}}^{m \dagger}(t)\right]=-i \tilde{g}_{r^{m} s^{m}}^{m}
$$

This ensures that

$$
\frac{d}{d t}\left[\tilde{a}_{r^{m}}^{m}(t), \tilde{a}_{s^{m}}^{m \dagger}(t)\right]=0,
$$

i.e., if the modal basis is orthonormal at $t=0$, it will continue to be orthonormal at later times. This is analogous to requiring in first quantization $\left\langle\tilde{\phi}_{r^{m}}^{m} \mid \dot{\tilde{\phi}}_{s^{m}}^{m}\right\rangle=-i\left\langle\tilde{\phi}_{r^{m}}^{m}\left|g^{m}\right| \tilde{\phi}_{s^{m}}^{m}\right\rangle$ as employed in, e.g., Ref. 8 in the context of MCTDH.

An arbitrary variation to the wave function can be written as

$$
|\delta \tilde{\Phi}\rangle=\delta \tilde{a}_{i^{m}}^{m \dagger} \prod_{m^{\prime} \neq m} \tilde{a}_{i^{m^{\prime}}}^{m^{\prime} \dagger}|\mathrm{vac}\rangle .
$$

Choosing the variation to be orthogonal to the wave function itself $\left\langle\delta^{\perp} \tilde{\Phi} \mid \tilde{\Phi}\right\rangle=0$ requires

$$
\left[\delta^{\perp} \tilde{a}_{i^{m}}^{m}, \tilde{a}_{i^{m}}^{m \dagger}\right]=0 .
$$

Inserting the variation (35) with orthogonal one-mode variations into Eq. (6) results in

$$
\begin{aligned}
0= & \left\langle\operatorname{vac}\left|\delta^{\perp} \tilde{a}_{i^{m}}^{m} \prod_{m^{\prime} \neq m} \tilde{a}_{i^{m^{\prime}}}^{m^{\prime}} H \prod_{m^{\prime \prime}} \tilde{a}_{i^{m^{\prime \prime}}}^{m^{\prime \prime} \dagger}\right| \mathrm{vac}\right\rangle \\
& -i\left\langle\operatorname{vac}\left|\delta^{\perp} \tilde{a}_{i^{m}}^{m} \dot{\tilde{a}}_{i^{m}}^{m \dagger}\right| \mathrm{vac}\right\rangle .
\end{aligned}
$$


The orthogonal variation can be written as

$$
\langle\operatorname{vac}| \delta^{\perp} \tilde{a}_{i^{m}}^{m}=\langle\operatorname{vac}| \delta \tilde{a}_{i^{m}}^{m} O_{i^{m}}^{m}=\langle\operatorname{vac}| \delta \tilde{a}_{i^{m}}^{m} \sum_{a^{m}} \tilde{a}_{a^{m}}^{m \dagger} \tilde{a}_{a^{m}}^{m},
$$

using the orthogonal-projection operator of Eq. (31). Inserting (38) into (37) and using (33) gives

$$
\begin{aligned}
i\left\langle\operatorname{vac}\left|\left[\delta \tilde{a}_{i^{m}}^{m}, \dot{\tilde{a}}_{i^{m}}^{m \dagger}\right]\right| \mathrm{vac}\right\rangle= & \sum_{a^{m}}\langle\operatorname{vac}|\left[\delta \tilde{a}_{i^{m}}^{m}, \tilde{a}_{a^{m}}^{m \dagger}\right] \tilde{a}_{a^{m}}^{m} \\
& \times \prod_{m^{\prime} \neq m} \tilde{a}_{i^{m^{\prime}}}^{m^{\prime}} H \prod_{m^{\prime \prime}} \tilde{a}_{i^{m^{\prime \prime}}}^{m^{\prime \prime} \dagger}|\mathrm{vac}\rangle \\
& +\tilde{g}_{i^{m} i^{m}}^{m}\left\langle\operatorname{vac}\left|\left[\delta \tilde{a}_{i^{m}}^{m}, \tilde{a}_{i^{m}}^{m \dagger}\right]\right| \mathrm{vac}\right\rangle .
\end{aligned}
$$

Using the fact that commutators between elementary operators are scalars (see Sec. II B 1) results in the following EOM for the time evolution of the occupied modals:

$$
i\left[\delta \tilde{a}_{i^{m}}^{m}, \dot{\tilde{a}}_{i^{m}}^{m \dagger}\right]=\sum_{a^{m}}\left[\delta \tilde{a}_{i^{m}}^{m}, \tilde{a}_{a^{m}}^{m \dagger}\right] \tilde{F}_{a^{m} i^{m}}^{m \tilde{\mathbf{i}}}+\tilde{g}_{i^{m} i^{m}}^{m}\left[\delta \tilde{a}_{i^{m}}^{m}, \tilde{a}_{i^{m}}^{m^{\dagger}}\right]
$$

where the mean-field operator $F^{m, \tilde{\mathbf{i}}}$ is the VSCF Fock operator (see Sec. III A). Note that the first term on the right-hand side of Eq. (40) is zero for all parallel variations where $\delta \tilde{a}_{i^{m}}^{m}=k \cdot \tilde{a}_{i^{m}}^{m}$, while the second term vanishes for orthogonal variations as defined in (36).

The time evolution of the phase is determined by (4) which results in

$$
\dot{F}=\langle\tilde{\Phi}|H| \tilde{\Phi}\rangle-\sum_{m=1}^{M} \tilde{g}_{i^{m} i^{m}}^{m} .
$$

\section{A. The mean-field operator}

The mean-field operator is a one-mode operator which can be expressed in both the time-dependent and the timeindependent bases as

$$
F^{m, \tilde{\mathbf{i}}}=\sum_{r^{m} s^{m}} \tilde{F}_{r^{m} s^{m}}^{m, \tilde{\mathbf{i}}} \tilde{a}_{r^{m}}^{m \dagger} \tilde{a}_{s^{m}}^{m}=\sum_{r^{m} s^{m}} F_{r^{m} s^{m}}^{m \tilde{\mathbf{i}}} a_{r^{m}}^{m \dagger} a_{s^{m}}^{m},
$$

where

$$
\tilde{F}_{r^{m} s^{m}}^{m, \tilde{\mathbf{i}}}=\left\langle\tilde{\Phi}\left|\left[\left[\tilde{a}_{r^{m}}^{m}, H\right], \tilde{a}_{s^{m}}^{m \dagger}\right]\right| \tilde{\Phi}\right\rangle
$$

and

$$
F_{r^{m} s^{m}}^{m \tilde{\mathbf{i}}}=\left\langle\tilde{\Phi}\left|\left[\left[a_{r^{m}}^{m}, H\right], a_{s^{m}}^{m \dagger}\right]\right| \tilde{\Phi}\right\rangle .
$$

The $\tilde{\mathbf{i}}$ superscript indicates that the integration over modes in the mean-field-operator elements is carried out using the timedependent wave function $|\tilde{\Phi}\rangle$.

It is evident from (43) that terms in the Hamiltonian which contain no reference to mode $m$ do not contribute to the mean-field operator. This observation allows for introducing an active-term algorithm as also used in VSCF, ${ }^{6}$

$$
\begin{aligned}
\tilde{F}_{r^{\prime} s^{m}}^{m \tilde{\mathbf{i}}} & =\sum_{t} c_{t}\left\langle\tilde{\Phi}\left|\left[\left[\tilde{a}_{r^{m}}^{m}, \prod_{m^{\prime} \in \mathbf{m}_{t}} \tilde{h}^{m^{\prime} t}\right], \tilde{a}_{s^{m}}^{m \dagger}\right]\right| \tilde{\Phi}\right\rangle \\
& =\sum_{t \in\left\{t_{\text {act }(m)\}}\right.} c_{t}\left\langle\tilde{\Phi}\left|\left[\left[\tilde{a}_{r^{m}}^{m}, \tilde{h}^{m t}\right], \tilde{a}_{s^{m}}^{m \dagger}\right] \prod_{m^{\prime} \in \mathbf{m}_{t} \backslash m} \tilde{h}^{m^{\prime} t}\right| \tilde{\Phi}\right\rangle \\
& =\sum_{t \in\left\{t_{\text {act }(m)\}}\right.}\left(c_{t} \prod_{m^{\prime} \in \mathbf{m}_{t} \backslash m} \tilde{h}_{i^{m^{\prime}} i^{m^{\prime}}}^{m^{\prime} t}\right) \tilde{h}_{r^{m} s^{m}}^{m t}=\sum_{t \in\left\{t_{\text {act }(m)\}}\right.} \tilde{z}^{m t} \tilde{h}_{r^{m} s^{m}}^{m t},
\end{aligned}
$$

where $\left\{t_{\mathrm{act}(m)}\right\}$ contains only terms that operate on mode $m$. Also note that

$$
F_{r^{m} S^{m}}^{m, \tilde{\mathbf{i}}}=\sum_{t \in\left\{t_{\text {act }(m)}\right\}} \tilde{z}^{m t} h_{r^{m} S^{m}}^{m t}
$$

depends on the time-dependent $\tilde{z}^{m t}$; i.e., the integrals of the mean-field operator always depend on the time-dependent basis.

The analysis in Ref. 6 shows that removing inactive terms from the mean-field operator in VSCF corresponds to a reduction of the computational complexity with respect to the number of modes in the system $(M)$ by one order. For example, if the Hamiltonian contains only two-mode couplings, the computational scaling with the number of degrees of freedom is reduced from $M^{3}$ down to $M^{2}$. In addition, it was found that for large molecules, some interactions were so weak that screening techniques could lower the computational scaling in VSCF even further, eventually down to linear in the uncoupled case. While this computational scaling may seem intuitive given the only linear increase in the number of parameters, it is difficult to achieve in an actual numerical implementation with Hamiltonian operators containing non-trivial couplings. Nevertheless, we shall continue to draw benefit from the VSCF experience in this work. It is important to utilize active terms in order to obtain low $M$ scaling. As discussed in Ref. 6, the partitioning of the mean-field operator into active and inactive terms can also be realized in first quantization. We do not know if this has been done in other TDH implementations, but it is included in both the algorithms we implement in the following.

\section{B. Linear parametrization}

If we choose to expand the time-dependent modals in an orthogonal, time-independent basis defined by the creation and annihilation operators $\left\{a_{q^{m}}^{m \dagger}\right\}$ and $\left\{a_{p^{m}}^{m}\right\}$ satisfying the usual commutation relations, we define the L-TDH method by writing the time-dependent operators as

$$
\begin{aligned}
& \tilde{a}_{r^{m}}^{m \dagger}(t)=\sum_{q^{m}} a_{q^{m}}^{m \dagger} C_{q^{m} r^{m}}^{m}(t), \\
& \tilde{a}_{S^{m}}^{m}(t)=\sum_{p^{m}} a_{p^{m}}^{m} C_{p^{m} S^{m}}^{m *}(t),
\end{aligned}
$$

where the $\mathbf{C}^{m}$ matrices are the L-TDH time-dependent modal coefficients which are chosen to be unitary at $t=0$ in order to fulfill (34).

Inserting these definitions into (40) results in

$$
\begin{aligned}
0= & \sum_{p^{m}} \delta C_{p^{m^{m}}}^{m *}\left(i \sum_{q^{m}} \dot{C}_{q^{m} i^{m}}^{m}\left[a_{p^{m}}^{m}, a_{q^{m}}^{m \dagger}\right]-\sum_{a^{m}} \tilde{F}_{a^{m} i^{m}}^{m \tilde{\tilde{\mathbf{i}}}}\right. \\
& \left.\times \sum_{q^{m}} C_{q^{m} a^{m}}^{m}\left[a_{p^{m}}^{m}, a_{q^{m}}^{m^{\dagger}}\right]-\tilde{g}_{i^{m} i^{m}}^{m} \sum_{q^{m}} C_{q^{m} i^{m}}^{m}\left[a_{p^{m}}^{m}, a_{q^{m}}^{m^{\dagger}}\right]\right) \\
= & \sum_{p^{m}} \delta C_{p^{m} i^{m}}^{m *}\left(i \dot{C}_{p^{m} i^{m}}^{m}-\sum_{a^{m}} C_{p^{m}}^{m} a^{m} \tilde{F}_{a^{m} i^{m}}^{m \tilde{\mathbf{i}}}-\tilde{g}_{i^{m} i^{m}}^{m} C_{p^{m} i^{m}}^{m}\right),
\end{aligned}
$$

which for arbitrary variations of the $C^{m}$-matrix elements gives 


$$
i \dot{C}_{p^{m} i^{m}}^{m}=\sum_{a^{m}} C_{p^{m} a^{m}}^{m} \tilde{F}_{a^{m} i^{m}}^{m \tilde{\mathbf{i}}}+C_{p^{m} i^{m}}^{m} \tilde{g}_{i^{m} i^{m}}^{m}
$$

Note that the variations of the $C^{m}$-matrix elements are in principle arbitrary, but the constraint operator $g^{m}$ ensures that the time evolution conserves the orthonormality of the onemode functions. Thereby, the $\mathbf{C}^{m}$ matrices stay unitary at all times.

Equation (50) contains reference to the expansion coefficients of the virtual modals $C_{p^{m} a^{m}}^{m}$ which are simply defined as the orthogonal complement to the occupied modals. Instead of explicitly constructing the orthogonal complement, the L-TDH EOMs can be written in terms of the time-independent basis as

$$
\begin{aligned}
i \dot{C}_{p^{m} i^{m}}^{m} & =\sum_{q^{m}}\left[F_{p^{m} q^{m}}^{m \tilde{\mathbf{i}}}-\delta_{p^{m} q^{m}}\left(\tilde{F}_{i^{m} i^{m}}^{m \tilde{\mathbf{i}}}-\tilde{g}_{i^{m} i^{m}}^{m}\right)\right] C_{q^{m} i^{m}}^{m} \\
& =\sum_{q^{m}} h_{p^{m} q^{m}}^{\mathrm{eff}, m} C_{q^{m} i^{m}}^{m},
\end{aligned}
$$

written in the matrix form as

$$
i \dot{\mathbf{C}}^{m}=\mathbf{h}^{\text {eff, }, m} \mathbf{C}^{m} .
$$

Note that the $\tilde{F}_{i^{m} i^{m}}^{m \tilde{\mathbf{i}}}$ matrix element of the mean-field operator (for any $m$ ) is equal to the energy $\langle\tilde{\Phi}|H| \tilde{\Phi}\rangle$ apart from inactive terms not included in the mean-field operator.

The final working equations are obtained by choosing a specific constraint operator $g^{m}$, and we mention a few choices here. The obvious choice $g^{m}=0$ results in the following equations of motion:

$$
\begin{gathered}
i \dot{C}_{p^{m} i^{m}}^{m}=\sum_{q^{m}}\left[F_{p^{m} q^{m}}^{m, \tilde{\mathbf{i}}}-\delta_{p^{m} q^{m}} \tilde{F}_{i^{m} i^{m}}^{m \tilde{\mathbf{i}}}\right] C_{q^{m} i^{m}}^{m}, \\
\dot{F}=\langle\tilde{\Phi}|H| \tilde{\Phi}\rangle .
\end{gathered}
$$

Choosing $g^{m}=F^{m, \tilde{\mathbf{i}}}$, we obtain instead

$$
\begin{gathered}
i \dot{C}_{p^{m} i^{m}}^{m}=\sum_{q^{m}} F_{p^{m} q^{m}}^{m, \tilde{\mathbf{i}}} C_{q^{m} i^{m}}^{m}, \\
\dot{F}=\langle\tilde{\Phi}|H| \tilde{\Phi}\rangle(1-M),
\end{gathered}
$$

and thereby $h^{\mathrm{eff}, m}=F^{m, \tilde{\mathbf{i}}}$.

\section{TDH IN EXPONENTIAL PARAMETRIZATION}

\section{A. Derivation of equations of motion}

We now derive the EOMs for an exponentially parametrized state of the form

$$
|\tilde{\Phi}\rangle=\exp (\kappa)|\Phi\rangle,
$$

where $|\Phi\rangle$ is a time-independent reference state. EOMs can be derived from Eq. (40) using the time-dependent creation and annihilation operators defined in Eq. (22). However, in order to cover exact states as well as TDH, we derive EOMs for a general single-exponential ansatz.

We adopt a notation similar to the standard notation in the response-function community and, excluding the redundant parameters, we define here the vectors of excitation parameters,

$$
\left(\boldsymbol{\kappa}_{+}^{m}\right)_{a^{m}}=\kappa_{a^{m} i^{m}}^{m},
$$

together with the vector of de-excitation parameters,

$$
\left(\boldsymbol{\kappa}_{-}^{m}\right)_{a^{m}}=-\kappa_{a^{m} i^{m}}^{m *}
$$

and the full vectors of parameters for the mode $m$,

$$
\boldsymbol{\kappa}^{m}=\left(\begin{array}{c}
\boldsymbol{\kappa}_{+}^{m} \\
\mathbf{\kappa}_{-}^{m}
\end{array}\right),
$$

and the corresponding time-derivatives,

$$
\dot{\mathbf{\kappa}}^{m}=\left(\begin{array}{c}
\dot{\mathbf{\kappa}}_{+}^{m} \\
\dot{\mathbf{\kappa}}_{-}^{m}
\end{array}\right) \text {, }
$$

so that all the objects depending on $\kappa$ can also be seen as depending on the $m \mathbf{\kappa}^{m}$ vectors.

The mode- $m$ part of the $\kappa$ operator is then written as

$$
\kappa^{m}=\mathbf{v}^{m T} \boldsymbol{\kappa}^{m}=\boldsymbol{\kappa}^{m T} \mathbf{v}^{m},
$$

where $\mathbf{v}^{m}$ is a vector of shift operators (or state-transfer operators in the VCI limit). For later reference, we also define the vector $\mathbf{u}^{m}=\left[\kappa^{m}, \mathbf{v}^{m}\right]$,

$$
\mathbf{v}^{m} \equiv\left(\begin{array}{c}
\mathbf{v}_{+}^{m} \\
\mathbf{v}_{-}^{m}
\end{array}\right), \quad \mathbf{u}^{m} \equiv\left(\begin{array}{c}
\mathbf{u}_{+}^{m} \\
\mathbf{u}_{-}^{m}
\end{array}\right) \equiv\left[\kappa^{m}, \mathbf{v}^{m}\right],
$$

with elements defined as

$$
\begin{aligned}
& {\left[\mathbf{v}_{+}^{m}\right]_{a^{m}} \equiv E_{a^{m} i^{m}}^{m},} \\
& {\left[\mathbf{v}_{-}^{m}\right]_{a^{m}} \equiv E_{i^{m} a^{m}}^{m}} \\
& {\left[\mathbf{u}_{+}^{m}\right]_{a^{m}} \equiv\left[\kappa^{m},\left[\mathbf{v}_{+}^{m}\right]_{a^{m}}\right]=-\kappa_{a^{m} i^{m}}^{m *} E_{i^{m} i^{m}}^{m}+\sum_{b^{m}} \kappa_{b^{m} i^{m}}^{m *} E_{a^{m} b^{m}}^{m},} \\
& {\left[\mathbf{u}_{-}^{m}\right]_{a^{m}} \equiv\left[\kappa^{m},\left[\mathbf{v}_{-}^{m}\right]_{a^{m}}\right]=-\kappa_{a^{m} i^{m}}^{m} E_{i^{m} i^{m}}^{m}+\sum_{b^{m}} \kappa_{b^{m} i^{m}}^{m} E_{b^{m} a^{m}}^{m} .}
\end{aligned}
$$

Note that $\mathbf{v}_{+}^{\dagger}=\mathbf{v}_{-}^{T}$ and $\boldsymbol{\kappa}_{+}^{m \dagger}=-\boldsymbol{\kappa}_{-}^{m T}$ which ensures that $\kappa$ is an anti-Hermitian operator.

We define commutators between the operator vectors as matrices with elements $[\mathbf{a}, \mathbf{b}]_{i j}=\left[a_{i}, b_{j}\right]$,

$$
\left[\mathbf{v}_{+}^{m}, \mathbf{v}_{+}^{m}\right]=\left[\mathbf{v}_{-}^{m}, \mathbf{v}_{-}^{m}\right]=\mathbf{0},
$$

$$
\left[\mathbf{v}_{+}^{m}, \mathbf{v}_{-}^{m}\right]=\mathbf{E}_{v}^{m}-v_{0}^{m} \mathbf{1},
$$

with $\left[\mathbf{E}_{v}^{m}\right]_{a^{m} b^{m}}=E_{a^{m} b^{m}}^{m}$ and $v_{0}^{m}=E_{i^{m} i^{m}}^{m}$. Note that $\mathbf{E}_{v}^{m}|\Phi\rangle=\mathbf{0}$ and $v_{0}^{m}|\Phi\rangle=|\Phi\rangle$.

We now define the similarity-transformed time derivative,

$$
\mathcal{D}=e^{-\kappa}\left(\frac{\partial e^{\kappa}}{\partial t}\right)=\int_{0}^{1} e^{-s \kappa} \dot{\kappa} e^{s \kappa} d s=\sum_{n=0}^{\infty} \frac{(-1)^{n}}{(n+1) !}\left(\kappa_{[}\right)^{n} \dot{\kappa},
$$

where we have used the relation for derivatives of exponential operators $^{34}$ as well as the Baker-Campbell-Hausdorff (BCH) expansion and defined the $n$th nested commutator with the $\kappa$ operator as $\left(\kappa_{[}\right)^{n}$, e.g., $\left(\kappa_{[}\right)^{2} \Omega=[\kappa,[\kappa, \Omega]]$. The $\mathcal{D}$ operator can be partitioned into terms for each mode, 


$$
\mathcal{D}=\sum_{m} e^{-\kappa^{m}}\left(\frac{\partial e^{\kappa^{m}}}{\partial t}\right)=\sum_{m} \mathcal{D}_{m},
$$

and each one-mode operator can be split into even and odd contributions,

$$
\begin{aligned}
\mathcal{D}_{m}= & \sum_{n=0}^{\infty} \frac{1}{(2 n+1) !}\left(\kappa_{[}^{m}\right)^{2 n} \dot{\kappa}^{m} \\
& +\sum_{n=0}^{\infty} \frac{-1}{(2 n+2) !}\left(\kappa_{[}^{m}\right)^{2 n+1} \dot{\kappa}^{m}=\mathcal{D}_{m}^{E}+\mathcal{D}_{m}^{O} .
\end{aligned}
$$

As shown later in Secs. IV C and IV D, the two contributions can be written as

$$
\mathcal{D}_{m}^{E}=\mathbf{v}^{m T} \mathbf{D}_{m}^{E} \dot{\boldsymbol{\kappa}}^{m}, \quad \mathcal{D}_{m}^{O}=\mathbf{u}^{m T} \mathbf{D}_{m}^{O} \dot{\boldsymbol{\kappa}}^{m},
$$

where $\mathbf{D}_{m}^{E}$ and $\mathbf{D}_{m}^{O}$ are Hermitian matrices. We shall see that only the even terms contribute to the TDH EOMs for the coefficients, and, conversely, only the odd terms contribute to the phase equation.

Equations of motion for the $\kappa$ parameters are obtained from the TDVP [Eq. (5)] using the following variation, obtained with analogous considerations for the derivatives with respect to parameters as carried out above for the time-derivatives:

$$
\begin{aligned}
|\delta \tilde{\Phi}\rangle & =\sum_{m} \sum_{r^{m} s^{m}} \delta \kappa_{r^{m} s^{m}}^{m}\left(\frac{\partial e^{\kappa}}{\partial \kappa_{r^{m} s^{m}}^{m}}\right)|\Phi\rangle \\
& =\sum_{m} \sum_{r^{m} s^{m}} \delta \kappa_{r^{m} s^{m}}^{m} e^{\kappa}\left(\frac{\partial \mathcal{D}}{\partial \dot{\kappa}_{r^{m} s^{m}}^{m}}\right)|\Phi\rangle \\
& =e^{\kappa} \sum_{m}\left(\mathbf{v}^{m T} \mathbf{D}_{m}^{E}+\mathbf{u}^{m T} \mathbf{D}_{m}^{O}\right) \delta \boldsymbol{\kappa}^{m}|\Phi\rangle=e^{\kappa} \delta \bar{\kappa}|\Phi\rangle .
\end{aligned}
$$

Here the $\delta \bar{\kappa}$ operator has been implicitly defined from the vectors of variations $\left\{\delta \boldsymbol{\kappa}^{m}\right\}$ and the $\mathbf{D}$ matrices. This results in

$$
\begin{aligned}
0 & =\operatorname{Re}\left\langle\Phi\left|\delta \bar{\kappa}^{\dagger} e^{-\kappa}\left(H-i \frac{\partial}{\partial t}\right) e^{\kappa}\right| \Phi\right\rangle \\
& =\operatorname{Re}\left\langle\Phi\left|\delta \bar{\kappa}^{\dagger}\left(e^{-\kappa} H e^{\kappa}-i \mathcal{D}\right)\right| \Phi\right\rangle \\
& =\frac{1}{2}\left\langle\Phi\left|\left[\delta \bar{\kappa}^{\dagger}, e^{-\kappa} H e^{\kappa}-i \mathcal{D}\right]\right| \Phi\right\rangle \\
& =\frac{1}{2} \sum_{m} \delta \mathbf{\kappa}^{m \dagger} \mathbf{D}_{m}^{E}\left\langle\Phi\left|\left[\left(\mathbf{v}^{m T}\right)^{\dagger}, e^{-\kappa} H e^{\kappa}-i \mathcal{D}\right]\right| \Phi\right\rangle,
\end{aligned}
$$

where we have used the fact that the odd term in $\delta \bar{\kappa}$ cancels out since it only contains $E_{i^{m} i^{m}}^{m}$ and $E_{a^{m} b^{m}}^{m}$ operators. For arbitrary variations, we obtain

$$
\mathbf{0}=\mathbf{D}_{m}^{E}\left(\left\langle\Phi\left|\left[\mathbf{v}_{s}^{m}, e^{-\kappa^{m}} H e^{\kappa^{m}}\right]\right| \Phi\right\rangle-i\left\langle\Phi\left|\left[\mathbf{v}_{s}^{m}, \mathcal{D}_{m}^{E}\right]\right| \Phi\right\rangle\right),
$$

where $\mathbf{v}_{s}^{m T}=\left(\mathbf{v}_{-}^{m T} \mathbf{v}_{+}^{m T}\right)$. After a few manipulations, the final EOMs are obtained as

$$
\mathbf{D}_{m}^{E} \mathbf{S}\left(\mathbf{D}_{m}^{E} \dot{\boldsymbol{\kappa}}^{m}-\mathbf{b}^{m}\right)=\mathbf{0}
$$

where

$$
\mathbf{S} \equiv\left\langle\Phi\left|\left[\mathbf{v}_{s}^{m}, \mathbf{v}^{m}\right]\right| \Phi\right\rangle=\left(\begin{array}{rr}
\mathbf{1} & \mathbf{0} \\
\mathbf{0} & -\mathbf{1}
\end{array}\right)
$$

and

$$
\mathbf{b}^{m} \equiv\left(\begin{array}{c}
\mathbf{b}_{+}^{m} \\
\mathbf{b}_{-}^{m}
\end{array}\right)
$$

with

$$
\begin{aligned}
{\left[\mathbf{b}_{+}^{m}\right]_{a^{m}} } & =-i \tilde{F}_{a^{m} i^{m}}^{m, \tilde{\mathbf{i}}}, \\
{\left[\mathbf{b}_{-}^{m}\right]_{a^{m}} } & =-i \tilde{F}_{i^{m} a^{m}}^{m, \tilde{\mathbf{i}}} \quad\left(=-\left[\mathbf{b}_{+}^{m}\right]_{a^{m}}^{*}\right) .
\end{aligned}
$$

For the values of $\kappa$ where $\mathbf{D}_{m}^{E}$ is non-singular, Eq. (77) is equivalent to solving the EOMs,

$$
\mathbf{D}_{m}^{E} \dot{\boldsymbol{\kappa}}^{m}=\mathbf{b}^{m}
$$

for each mode. When $\mathbf{D}_{m}^{E}$ becomes singular (which happens at a well-defined set of points described later), Eq. (77) is trivially fulfilled and Eq. (82) cannot be used as it stands. This, however, can easily be handled in practice as discussed further in Sec. VI, and in Subsections IV B and IV C, the precise locations of the singularities are described.

The explicit structure of the $D$ matrices is derived in Secs. IV C and IV D, but first the exponential transformation is analyzed in more detail.

\section{B. Analysis of the exponential transformation}

Consider the matrix representation of a $\kappa^{m}$ operator in a single-mode modal basis,

$$
\mathbf{K}^{m}=\left(\begin{array}{cc}
\mathbf{0}_{1 \times 1} & \boldsymbol{\kappa}_{-}^{m T} \\
\boldsymbol{\kappa}_{+}^{m} & \mathbf{0}_{\left(N^{m}-1\right) \times\left(N^{m}-1\right)}
\end{array}\right),
$$

where there are $N^{m}$ basis functions for mode $m$ and therefore $N^{m}-1$ parameters in the $\boldsymbol{\kappa}_{+}^{m}$ column vector and the whole matrix is of dimensionality $N^{m} \times N^{m}$. We now introduce

$$
R_{m}=\sqrt{\left|\boldsymbol{\kappa}_{+}^{m}\right|^{2}}=\sqrt{-\boldsymbol{\kappa}_{-}^{m T} \boldsymbol{\kappa}_{+}^{m}}
$$

as well as $\gamma_{ \pm}^{m}$ vectors defined as having norm one and direction along the corresponding $\boldsymbol{\kappa}_{ \pm}^{m}$ vectors,

$$
\mathbf{\kappa}_{+}^{m}=R_{m} \gamma_{+}^{m}, \quad \mathbf{\kappa}_{-}^{m}=R_{m} \gamma_{-}^{m},
$$

and $\mathbf{K}^{m}=R_{m} \Gamma^{m}$ with

$$
\boldsymbol{\Gamma}^{m}=\left(\begin{array}{cc}
\mathbf{0}_{1 \times 1} & \boldsymbol{\gamma}_{-}^{m T} \\
\boldsymbol{\gamma}_{+}^{m} & \mathbf{0}_{\left(N^{m}-1\right) \times\left(N^{m}-1\right)}
\end{array}\right) .
$$

This particular form of the matrix allows for an easy evaluation of the matrix representation of the exponential operator $\exp (\kappa)$ since the powers of the $\boldsymbol{\Gamma}^{m}$ matrix can be calculated as a blockwise product,

$$
\begin{aligned}
& \left(\boldsymbol{\Gamma}^{m}\right)^{2}=\left(\begin{array}{cc}
-1 & \mathbf{0}_{1 \times\left(N^{m}-1\right)} \\
\mathbf{0}_{\left(N^{m}-1\right) \times 1} & \boldsymbol{\gamma}_{+}^{m} \boldsymbol{\gamma}_{-}^{m T}
\end{array}\right), \\
& \left(\boldsymbol{\Gamma}^{m}\right)^{3}=\left(\begin{array}{cc}
\mathbf{0}_{1 \times 1} & -\boldsymbol{\gamma}_{-}^{m T} \\
-\boldsymbol{\gamma}_{+}^{m} & \mathbf{0}_{\left(N^{m}-1\right) \times\left(N^{m}-1\right)}
\end{array}\right)=-\boldsymbol{\Gamma}^{m} .
\end{aligned}
$$

In general, one has

$$
\begin{aligned}
\left(\mathbf{K}^{m}\right)^{2 n} & = \begin{cases}\mathbf{1} & \text { for } n=0 \\
(-1)^{n-1} R_{m}^{2 n}\left(\boldsymbol{\Gamma}^{m}\right)^{2} & \text { for } n>0,\end{cases} \\
\left(\mathbf{K}^{m}\right)^{2 n+1} & =(-1)^{n} R_{m}^{2 n+1} \boldsymbol{\Gamma}^{m} .
\end{aligned}
$$


Separating even and odd terms in the series expansion of $\exp \left(\mathbf{K}^{m}\right)$ and using standard Taylor series, we obtain

$$
\begin{aligned}
\exp \left(\mathbf{K}^{m}\right) & =\mathbf{1}+\sin \left(R_{m}\right) \boldsymbol{\Gamma}^{m}+\left(1-\cos \left(R_{m}\right)\right)\left(\boldsymbol{\Gamma}^{m}\right)^{2} \\
& =\mathbf{1}+\operatorname{sinc}\left(R_{m}\right) \mathbf{K}^{m}+\frac{1}{2} \operatorname{sinc}^{2}\left(R_{m} / 2\right)\left(\mathbf{K}^{m}\right)^{2},
\end{aligned}
$$

where we have used $1-\cos (x)=2 \sin ^{2}(x / 2)$ as well as the unnormalized sinc function. It is evident that $\exp \left(\mathbf{K}^{m}\right)$ is periodic in $R_{m}$, with period $2 \pi$, so that values of $\mathbf{K}^{m}$ corresponding to the same $\boldsymbol{\Gamma}^{m}$ can give the same exponential. Note that the $\gamma_{ \pm}^{m}$ vectors are not defined when $\boldsymbol{\kappa}^{m}=\mathbf{0}$, i.e., $R_{m}=0$. In that case, however, $\exp \left(\mathbf{K}^{m}\right)=\mathbf{1}$ in agreement with the corresponding limit $\left(R_{m} \rightarrow 0\right)$ of Eq. (91).

Using (91), the following expression for the timedependent creation operators is obtained:

$$
\tilde{a}_{i^{m}}^{m \dagger}=\cos \left(R_{m}\right) a_{i^{m}}^{m \dagger}+\operatorname{sinc}\left(R_{m}\right) \sum_{a^{m}} a_{a^{m}}^{m \dagger} \kappa_{a^{m} i^{m}}^{m},
$$

which relates the linear and exponential parametrizations. Note that the weight of $a_{i^{m}}^{m \dagger}$ in $\tilde{a}_{i^{m}}^{m \dagger}$ is zero for $R_{m}=\left(n+\frac{1}{2}\right) \pi$ and $\tilde{a}_{i^{m}}^{m \dagger}= \pm a_{i^{m}}^{m \dagger}$ for $R_{m}=n \pi$. These are the points (except $R_{m}=0$ ) where the $\mathbf{D}_{m}^{E}$ matrix becomes singular as seen in Subsection IV C.

\section{Analysis of the $\mathcal{D}^{E}$ operator}

In order to calculate the nested commutators of Eq. (70) in a convenient way, we first define $D_{m}^{(n)}=\left(\kappa_{[}^{m}\right)^{n} \dot{\kappa}^{m}$ and write

$$
\begin{aligned}
& D_{m}^{(0)}=\dot{\kappa}^{m}=\mathbf{v}^{m T} \dot{\boldsymbol{\kappa}}^{m}, \\
& D_{m}^{(1)}=\left[\kappa^{m}, D_{m}^{(0)}\right]=\left[\kappa^{m}, \dot{\kappa}^{m}\right]=\mathbf{u}^{m T} \dot{\boldsymbol{\kappa}}^{m} .
\end{aligned}
$$

Explicit evaluation of the commutators gives after some manipulations

$$
\left[\kappa^{m}, \mathbf{u}^{m T}\right]=\left[\kappa^{m},\left[\kappa^{m}, \mathbf{v}^{m T}\right]\right]=\mathbf{v}^{m T} \mathbf{C}_{m},
$$

where we have introduced the $2\left(N^{m}-1\right) \times 2\left(N^{m}-1\right)$ matrix $\mathbf{C}_{m}$ as

$$
\mathbf{C}_{m}=\left(\begin{array}{cc}
\boldsymbol{\kappa}_{+}^{m} \boldsymbol{\kappa}_{-}^{m T} & -2 \boldsymbol{\kappa}_{+}^{m} \boldsymbol{\kappa}_{+}^{m T} \\
-2 \boldsymbol{\kappa}_{-}^{m} \boldsymbol{\kappa}_{-}^{m T} & \boldsymbol{\kappa}_{-}^{m} \boldsymbol{\kappa}_{+}^{m T}
\end{array}\right)-R_{m}^{2} \mathbf{1} .
$$

Here 1 is the $2\left(N^{m}-1\right) \times 2\left(N^{m}-1\right)$ identity matrix recalling $2\left(N^{m}-1\right)$ as the number of $\kappa$ coefficients for mode $m$.

With (93) and (94) as the base cases and using (95) in the inductive step, it is seen by induction that we generally have, for all $n \in \mathbb{N}_{0}$,

$$
\begin{aligned}
D_{m}^{(2 n)} & =\mathbf{v}^{m T} \mathbf{C}_{m}^{n} \dot{\boldsymbol{\kappa}}^{m}, \\
D_{m}^{(2 n+1)} & =\mathbf{u}^{m T} \mathbf{C}_{m}^{n} \dot{\boldsymbol{\kappa}}^{m} .
\end{aligned}
$$

When $R_{m} \equiv\left\|\boldsymbol{\kappa}_{ \pm}^{m}\right\| \neq 0, \boldsymbol{\gamma}_{ \pm}^{m} \equiv \boldsymbol{\kappa}_{ \pm}^{m} / R_{m}$ is well defined, and we can thus proceed further by introducing the $2\left(N^{m}-1\right) \times 2$ dimensional $\mathbf{X}_{m}$ matrix

$$
\mathbf{X}_{m}=\left(\begin{array}{cc}
\gamma_{+}^{m} & \mathbf{0} \\
\mathbf{0} & \boldsymbol{\gamma}_{-}^{m}
\end{array}\right)
$$

and the $2 \times 2$ matrix (common to all modes)

$$
\mathbf{Y}=\left(\begin{array}{cc}
-1 & 2 \\
2 & -1
\end{array}\right)
$$

which allows us to rewrite $\mathbf{C}_{m}$ as

$$
\mathbf{C}_{m}=R_{m}^{2}\left(\mathbf{X}_{m} \mathbf{Y} \mathbf{X}_{m}^{\dagger}-\mathbf{1}\right) .
$$

The matrices $\mathbf{X}_{m}$ and $\mathbf{X}_{m}^{\dagger}$ are pseudoinverses of each other since they satisfy the four Penrose conditions: (i) $\mathbf{A A}^{+} \mathbf{A}=\mathbf{A}$; (ii) $\mathbf{A}^{+} \mathbf{A} \mathbf{A}^{+}=\mathbf{A}^{+}$; (iii) $\left(\mathbf{A} \mathbf{A}^{+}\right)^{\dagger}=\mathbf{A} \mathbf{A}^{+}$; (iv) $\left(\mathbf{A}^{+} \mathbf{A}\right)^{\dagger}=\mathbf{A}^{+} \mathbf{A}$. (Here $\mathbf{A}^{+}$is the pseudoinverse of $\mathbf{A}$ and $\mathbf{A}^{\dagger}$ is its Hermitian transpose. ${ }^{35}$ ) We can then define the orthogonal projector

$$
\mathbf{P}_{m}=\mathbf{X}_{m} \mathbf{X}_{m}^{\dagger},
$$

on the column space, $\operatorname{colsp}\left(\mathbf{X}_{m}\right)$, of $\mathbf{X}_{m}$, as well as the orthogonal projector

$$
\mathbf{Q}_{m}=\mathbf{1}-\mathbf{P}_{m},
$$

on its orthogonal complement, $\operatorname{colsp}\left(\mathbf{X}_{m}\right)^{\perp}$ [or equivalently the projector onto null $\left(\mathbf{X}_{m}^{\dagger}\right)$, the null space of $\mathbf{X}_{m}^{\dagger}$ ].

Due to the fact that $\mathbf{X}_{m}^{\dagger} \mathbf{X}_{m}=\mathbf{1}_{2 \times 2}$, any matrix $\mathbf{M}$ of the form $\mathbf{X}_{m} \mathbf{M}_{2 \times 2} \mathbf{X}_{m}^{\dagger}$ satisfies that

$$
\mathbf{M}=\mathbf{M} \mathbf{P}_{m}=\mathbf{P}_{m} \mathbf{M}=\mathbf{P}_{m} \mathbf{M} \mathbf{P}_{m},
$$

while

$$
\mathbf{0}=\mathbf{M} \mathbf{Q}_{m}=\mathbf{Q}_{m} \mathbf{M}
$$

$\mathbf{C}_{m}$ can therefore be decomposed into uncoupled terms for the $\operatorname{colsp}\left(\mathbf{X}_{m}\right)$ and $\operatorname{colsp}\left(\mathbf{X}_{m}\right)^{\perp}$ parts, respectively,

$$
\begin{aligned}
\mathbf{C}_{m} & =R_{m}^{2}\left(\mathbf{X}_{m}\left(\mathbf{Y}-\mathbf{1}_{2 \times 2}\right) \mathbf{X}_{m}^{\dagger}-\mathbf{Q}_{m}\right) \\
& =-R_{m}^{2}\left(\mathbf{P}_{m} \mathbf{X}_{m}\left(\mathbf{1}_{2 \times 2}-\mathbf{Y}\right) \mathbf{X}_{m}^{\dagger} \mathbf{P}_{m}+\mathbf{Q}_{m}\right) .
\end{aligned}
$$

Due to the orthogonality of the projectors, arbitrary powers of $\mathbf{C}_{m}$ can be written as

$$
\mathbf{C}_{m}^{n}=(-1)^{n} R_{m}^{2 n}\left(\mathbf{P}_{m} \mathbf{X}_{m}\left(\mathbf{1}_{2 \times 2}-\mathbf{Y}\right)^{n} \mathbf{X}_{m}^{\dagger} \mathbf{P}_{m}+\mathbf{Q}_{m}\right) .
$$

Using Eqs. (72) and (97), the $\mathcal{D}_{m}^{E}$ can then be written as

$$
\begin{aligned}
\mathcal{D}_{m}^{E} & \equiv \sum_{n=0}^{\infty} \frac{1}{(2 n+1) !} D_{m}^{(2 n)}=\mathbf{v}^{m T}\left[\sum_{n=0}^{\infty} \frac{1}{(2 n+1) !} \mathbf{C}_{m}^{n}\right] \dot{\boldsymbol{\kappa}}^{m} \\
& =\mathbf{v}^{m T} \mathbf{D}_{m}^{E} \dot{\boldsymbol{\kappa}}^{m},
\end{aligned}
$$

with

$$
\begin{aligned}
\mathbf{D}_{m}^{E}= & \mathbf{P}_{m} \mathbf{X}_{m}\left[\sum_{n=0}^{\infty} \frac{(-1)^{n}}{(2 n+1) !} R_{m}^{2 n}\left(\mathbf{1}_{2 \times 2}-\mathbf{Y}\right)^{n}\right] \mathbf{X}_{m}^{\dagger} \mathbf{P}_{m} \\
& +\left[\sum_{n=0}^{\infty} \frac{(-1)^{n}}{(2 n+1) !} R_{m}^{2 n}\right] \mathbf{Q}_{m} .
\end{aligned}
$$

The matrix

$$
\mathbf{1}_{2 \times 2}-\mathbf{Y}=\left(\begin{array}{cc}
2 & -2 \\
-2 & 2
\end{array}\right)
$$

is unitarily diagonalizable, $\mathbf{1}_{2 \times 2}-\mathbf{Y}=\mathbf{U} \operatorname{diag}(4,0) \mathbf{U}^{\dagger}$, where 


$$
\mathbf{U}=\frac{1}{\sqrt{2}}\left(\begin{array}{cc}
1 & 1 \\
-1 & 1
\end{array}\right)
$$

The required powers of the matrix are thus $\left(\mathbf{1}_{2 \times 2}-\mathbf{Y}\right)^{n}$ $=\mathbf{U} \operatorname{diag}\left(2^{2 n}, \delta_{0 n}\right) \mathbf{U}^{\dagger}$, and recognizing that the infinite series correspond to those of unnormalized sinc functions [i.e., $\operatorname{sinc}(x) \equiv \sin (x) / x$ for $x \neq 0$ ], we obtain the result

$$
\mathbf{D}_{m}^{E}=\mathbf{P}_{m} \mathbf{X}_{m} \mathbf{U}\left(\begin{array}{cc}
\operatorname{sinc}\left(2 R_{m}\right) & 0 \\
0 & 1
\end{array}\right) \mathbf{U}^{\dagger} \mathbf{X}_{m}^{\dagger} \mathbf{P}_{m}+\operatorname{sinc}\left(R_{m}\right) \mathbf{Q}_{m} .
$$

This expression for $\mathbf{D}_{m}^{E}$ has been derived under the assumption that $R_{m} \neq 0$. We see that since $\lim _{x \rightarrow 0} \operatorname{sinc}(x)=1$,

$$
\begin{aligned}
\lim _{R_{m} \rightarrow 0} \mathbf{D}_{m}^{E} & =\mathbf{P}_{m} \mathbf{X}_{m} \mathbf{U} \mathbf{1}_{2 \times 2} \mathbf{U}^{\dagger} \mathbf{X}_{m}^{\dagger} \mathbf{P}_{m}+\mathbf{Q}_{m} \\
& =\mathbf{P}_{m}+\mathbf{Q}_{m}=\mathbf{1},
\end{aligned}
$$

regardless of the limit $\lim _{R_{m} \rightarrow 0} \mathbf{X}_{m}$. Furthermore, notice that for $R_{m}=0$, i.e., $\kappa^{m}=0$, the $\mathcal{D}_{m}^{E}$ operator is equal to its first term, $\mathcal{D}_{m}^{E}=\dot{\kappa}^{m}=\mathbf{v}^{m T} \dot{\boldsymbol{\kappa}}^{m}$. Thus, defining $\mathbf{D}_{m}^{E}$ to be equal to its limiting value $\mathbf{1}$ for $R_{m}=0$ means that $\mathbf{D}_{m}^{E}$ is continuous as a function of $\boldsymbol{\kappa}^{m}$ and consistent with $\mathcal{D}_{m}^{E}$, also for $R_{m}=0$.

We can rewrite the projector in terms of two projections on vectors $\left(\gamma_{+}^{m \dagger}-\gamma_{-}^{m \dagger}\right)^{\dagger}$ and $\left(\gamma_{+}^{m \dagger} \gamma_{-}^{m \dagger}\right)^{\dagger}$,

$$
\mathbf{P}_{m}=\mathbf{X}_{m} \mathbf{X}_{m}^{\dagger}=\mathbf{X}_{m} \mathbf{U} \mathbf{1}_{2 \times 2} \mathbf{U}^{\dagger} \mathbf{X}_{m}^{\dagger}=\mathbf{P}_{m, 1}+\mathbf{P}_{m, 2},
$$

with

$$
\begin{aligned}
& \mathbf{P}_{m, 1}=\mathbf{X}_{m} \mathbf{U}\left(\begin{array}{ll}
1 & 0 \\
0 & 0
\end{array}\right) \mathbf{U}^{\dagger} \mathbf{X}_{m}^{\dagger}=\frac{1}{2}\left(\begin{array}{c}
\gamma_{+}^{m} \\
-\gamma_{-}^{m}
\end{array}\right)\left(\begin{array}{ll}
\gamma_{+}^{m, \dagger} & -\gamma_{-}^{m, \dagger}
\end{array}\right), \\
& \mathbf{P}_{m, 2}=\mathbf{X}_{m} \mathbf{U}\left(\begin{array}{ll}
0 & 0 \\
0 & 1
\end{array}\right) \mathbf{U}^{\dagger} \mathbf{X}_{m}^{\dagger}=\frac{1}{2}\left(\begin{array}{c}
\gamma_{+}^{m} \\
\gamma_{-}^{m}
\end{array}\right)\left(\begin{array}{ll}
\gamma_{+}^{m, \dagger} & \gamma_{-}^{m, \dagger}
\end{array}\right) .
\end{aligned}
$$

This means that we can obtain the $\mathbf{D}_{m}^{E}$ in a simple form as

$$
\mathbf{D}_{m}^{E}=\operatorname{sinc}\left(2 R_{m}\right) \mathbf{P}_{m, 1}+\mathbf{P}_{m, 2}+\operatorname{sinc}\left(R_{m}\right)\left(1-\mathbf{P}_{m, 1}-\mathbf{P}_{m, 2}\right) .
$$

It is clearly seen from the definition of the projectors that $\mathbf{D}_{m}^{E}$ is Hermitian. The structure of the matrix is

$$
\mathbf{D}_{m}^{E}=\left(\begin{array}{cc}
\mathbf{A} & \mathbf{B} \\
\mathbf{B}^{*} & \mathbf{A}^{*}
\end{array}\right),
$$

where $\mathbf{A}=\mathbf{A}^{\dagger}$ and $\mathbf{B}=\mathbf{B}^{T}$. This structure is similar to the $\mathbf{E}^{[2]}$ matrix arising in VSCF and VCI response theory. ${ }^{36}$

As stated in Sec. IV B, $\mathbf{D}_{m}^{E}$ is singular for $R_{m} \in \frac{\pi}{2} \mathbb{N} \backslash\{0\}$. However, its reduction to the identity matrix for $R_{m} \rightarrow 0$ means that it is always possible to change the modal basis in order to obtain $\boldsymbol{\kappa}^{m}=0$ when the $R_{m}$ related to the previous $\boldsymbol{\kappa}^{m}$ approach $\pi / 2$, and use the $\boldsymbol{\kappa}^{m} \rightarrow 0$ limit [Eq. (125)] to carry on the first step after the change of basis.

\section{Calculation of $\mathcal{D}^{O}$}

Using Eqs. (98) and (72), $\mathcal{D}_{m}^{O}$ can be rewritten as

$$
\mathcal{D}_{m}^{O}=\mathbf{u}^{m T} \mathbf{D}_{m}^{O} \dot{\boldsymbol{\kappa}}^{m},
$$

where

$$
\mathbf{D}_{m}^{O}=\sum_{n=0}^{\infty} \frac{-1}{(2 n+2) !} \mathbf{C}_{m}^{n}
$$

Calculations similar to those in Sec. IV C give

$$
\mathbf{D}_{m}^{O}=\mathbf{P}_{m} \mathbf{X}_{m} \mathbf{U}\left(\begin{array}{cc}
-\frac{1}{2} \operatorname{sinc}^{2}\left(R_{m}\right) & 0 \\
0 & -\frac{1}{2}
\end{array}\right) \mathbf{U}^{\dagger} \mathbf{X}_{m}^{\dagger} \mathbf{P}_{m}-\frac{1}{2} \operatorname{sinc}^{2}\left(\frac{1}{2} R_{m}\right) \mathbf{Q}_{m} .
$$

As for the $\mathbf{D}_{m}^{E}$ matrix, $\mathbf{D}_{m}^{O}$ is ill defined for $R_{m}=0$, but we see that $\lim _{R_{m} \rightarrow 0} \mathbf{D}_{m}^{O}=-\frac{1}{2} \mathbf{1}$. Defining the limit to be its value for $R_{m}=0$ makes $\mathbf{D}_{m}^{O}$ a continuous matrix function of $\boldsymbol{\kappa}^{m}$. Furthermore, this is consistent with the definition of the $\mathcal{D}_{m}^{O}$ operator, which equals zero when $\boldsymbol{\kappa}^{m}=\mathbf{0}$ (and thus $\mathbf{u}^{m}=\mathbf{0}$ ).

The $\mathcal{D}_{m}^{O}$ matrix elements that contribute to the time evolution of $F$ can then be evaluated as

$$
\begin{aligned}
\left\langle\Phi\left|\mathcal{D}_{m}^{O}\right| \Phi\right\rangle & =\left\langle i^{m}\left|\mathbf{u}^{m T}\right| i^{m}\right\rangle \mathbf{D}_{m}^{O} \dot{\boldsymbol{\kappa}}^{m}=\left(\boldsymbol{\kappa}_{-}^{m T}-\boldsymbol{\kappa}_{+}^{m T}\right) \mathbf{D}_{m}^{O} \dot{\boldsymbol{\kappa}}^{m} \\
& =-\left(\boldsymbol{\kappa}_{+}^{m \dagger}-\boldsymbol{\kappa}_{-}^{m \dagger}\right) \mathbf{D}_{m}^{O} \dot{\boldsymbol{\kappa}}^{m}
\end{aligned}
$$

by using the expressions for $\left[\mathbf{u}_{ \pm}^{m}\right]_{a^{m}}$ in (66) and (67) and noting that only $E_{i^{m} i^{m}}^{m}$ terms contribute.

Observing that

$$
\left(\boldsymbol{\kappa}_{+}^{m \dagger}-\boldsymbol{\kappa}_{-}^{m \dagger}\right) \mathbf{P}_{m, k}= \begin{cases}\left(\boldsymbol{\kappa}_{+}^{m \dagger}-\boldsymbol{\kappa}_{-}^{m \dagger}\right) & \text { for } k=1 \\ \mathbf{0} & \text { for } k=2,\end{cases}
$$

the only contribution stems from the $\mathbf{P}_{m, 1}$ term, yielding the following form of the phase equation:

$$
\begin{aligned}
\dot{F}= & \langle\tilde{\Phi}|H| \tilde{\Phi}\rangle-i\langle\Phi|\mathcal{D}| \Phi\rangle=\sum_{t} c_{t} \prod_{m} \tilde{h}_{i^{m} i^{m}}^{m t} \\
& -i \sum_{m}\left(-\frac{1}{2} \operatorname{sinc}^{2}\left(R_{m}\right)\right)(-1)\left(\boldsymbol{\kappa}_{+}^{m \dagger}-\boldsymbol{\kappa}_{-}^{m \dagger}\right)\left(\begin{array}{l}
\dot{\boldsymbol{\kappa}}_{+}^{m} \\
\dot{\boldsymbol{\kappa}}_{-}^{m}
\end{array}\right) .
\end{aligned}
$$

\section{E. The projected $\dot{\boldsymbol{\kappa}}^{m}$ equations}

When the $\mathbf{D}_{m}^{E}$ matrices are non-singular, the TDH state can be propagated by solving Eqs. (82) and (124). In the limit $\mathbf{K}^{m} \rightarrow \mathbf{0}$, Eq. (82) reduces to

$$
\dot{\mathbf{\kappa}}^{m}=\mathbf{b}^{m}(\mathbf{\kappa}=\mathbf{0}) .
$$

We note that diagonalization of the mean-field operators corresponds to a stationarity condition as expected. If the ground state is optimal relative to the Hamiltonian, we have a VSCF state satisfying the Brillouin theorem, $\tilde{F}_{a^{m} i^{m}}^{m \tilde{\mathbf{i}}}$ $=\left\langle\tilde{\Phi}\left|\left[\tilde{E}_{i^{m} a^{m}}^{m}, H\right]\right| \tilde{\Phi}\right\rangle=0$ and thus $\dot{\boldsymbol{\kappa}}=\mathbf{0}$ and $F(t)=\langle\tilde{\Phi}|H| \tilde{\Phi}\rangle t$, which is the expected result.

Equation (82) can be solved numerically. As it stands, it appears that $\mathbf{D}_{m}^{E}$ must be handled explicitly. However, from the preceding analysis, one can derive simple and concise expressions using projection techniques. Accordingly we separate out $\dot{\boldsymbol{\kappa}}^{m}$ into components 


$$
\begin{aligned}
\dot{\boldsymbol{\kappa}}^{m} & =\mathbf{P}_{m, 1} \dot{\boldsymbol{\kappa}}^{m}+\mathbf{P}_{m, 2} \dot{\boldsymbol{\kappa}}^{m}+\left(1-\mathbf{P}_{m, 1}-\mathbf{P}_{m, 2}\right) \dot{\boldsymbol{\kappa}}^{m} \\
& =\dot{\boldsymbol{\kappa}}_{P_{1}}^{m}+\dot{\boldsymbol{\kappa}}_{P_{2}}^{m}+\dot{\boldsymbol{\kappa}}_{Q}^{m} .
\end{aligned}
$$

Projecting now (82) with $\mathbf{P}_{m, 1}, \mathbf{P}_{m, 2}$, and $\mathbf{Q}_{m}$, using (117), and noticing that by the anti-Hermitian relations for $\boldsymbol{\gamma}_{ \pm}^{m}$ and $\mathbf{b}_{ \pm}^{m}$,

$$
\begin{aligned}
& \frac{1}{2}\left(\begin{array}{cc}
\boldsymbol{\gamma}_{+}^{m \dagger} & \mp \boldsymbol{\gamma}_{-}^{m \dagger}
\end{array}\right)\left(\begin{array}{l}
\mathbf{b}_{+}^{m} \\
\mathbf{b}_{-}^{m}
\end{array}\right)=\frac{1}{2}\left(\boldsymbol{\gamma}_{+}^{m \dagger} \mathbf{b}_{+}^{m} \mp\left(\boldsymbol{\gamma}_{+}^{m \dagger} \mathbf{b}_{+}^{m}\right)^{*}\right) \\
& =\left\{\begin{array}{cc}
i y_{m}^{\mathfrak{I}} & \left(-; \mathbf{P}_{m, 1}\right) \\
y_{m}^{\mathfrak{R}} & \left(+; \mathbf{P}_{m, 2}\right)
\end{array}\right.
\end{aligned}
$$

where we have defined

$$
y_{m}=y_{m}^{\mathfrak{R}}+i y_{m}^{\mathfrak{I}}=\gamma_{+}^{m \dagger} \mathbf{b}_{+}^{m},
$$

we obtain

$$
\begin{aligned}
& \operatorname{sinc}\left(2 R_{m}\right) \dot{\boldsymbol{\kappa}}_{P_{1}}^{m}=\mathbf{P}_{m, 1} \mathbf{b}^{m}=i y_{m}^{\mathfrak{J}}\left(\begin{array}{c}
\boldsymbol{\gamma}_{+}^{m} \\
-\boldsymbol{\gamma}_{-}^{m}
\end{array}\right), \\
& \dot{\boldsymbol{\kappa}}_{P_{2}}^{m}=\mathbf{P}_{m, 2} \mathbf{b}^{m}=y_{m}^{\Re}\left(\begin{array}{c}
\boldsymbol{\gamma}_{+}^{m} \\
\boldsymbol{\gamma}_{-}^{m}
\end{array}\right) \text {, } \\
& \operatorname{sinc}\left(R_{m}\right) \dot{\boldsymbol{\kappa}}_{Q}^{m}=\mathbf{Q}_{m} \mathbf{b}^{m}=\mathbf{b}^{m}-\left(\begin{array}{cc}
y_{m} & \boldsymbol{\gamma}_{+}^{m} \\
y_{m}^{*} & \boldsymbol{\gamma}_{-}^{m}
\end{array}\right) \text {. }
\end{aligned}
$$

We see that the structure of $\dot{\boldsymbol{\kappa}}$ preserves the anti-Hermitian property as it should. All in all, we can propagate the X-TDH state by calculating

$$
\begin{aligned}
\dot{\boldsymbol{\kappa}}_{+}^{m} & =\left[\frac{2 i y_{m}^{\mathfrak{J}}}{\sin \left(2 R_{m}\right)}+\frac{y_{m}^{\mathfrak{R}}}{R_{m}}-\frac{y_{m}}{\sin \left(R_{m}\right)}\right] \boldsymbol{\kappa}_{+}^{m}+\frac{1}{\operatorname{sinc}\left(R_{m}\right)} \mathbf{b}_{+}^{m} \\
& =\frac{1}{\sin \left(R_{m}\right)}\left[\left(\frac{i y_{m}^{\mathfrak{I}}}{\cos \left(R_{m}\right)}+\operatorname{sinc}\left(R_{m}\right) y_{m}^{\mathfrak{R}}-y_{m}\right) \mathbf{\kappa}_{+}^{m}+R_{m} \mathbf{b}_{+}^{m}\right] .
\end{aligned}
$$

The corresponding negative-index part can be calculated by complex conjugation, and in this manner, we only need to calculate the positive-index part of the mean-field vector $\mathbf{b}^{m}$. The EOM for the phase factor (124) can be rewritten as

$$
\dot{F}=\sum_{t} c_{t} \prod_{m} \tilde{h}_{i^{m} i^{m}}^{m t}+\sum_{m} \tan \left(R_{m}\right) y_{m}^{\mathfrak{J}},
$$

by using (129). Note that $\dot{F}$ is real in accord with the requirements set up earlier.

As has been seen before, (132) and also (133) appear to be ill defined for $R_{m}=0$, but fortunately the limits are, independent of $\lim _{R_{m} \rightarrow 0} \boldsymbol{\gamma}_{+}^{m}$,

$$
\begin{aligned}
\lim _{R_{m} \rightarrow 0} \dot{\boldsymbol{\kappa}}_{+}^{m} & =\underbrace{\left[i y_{m}^{\mathfrak{J}}+y_{m}^{\mathfrak{R}}-y_{m}\right]}_{=0} \gamma_{+}^{m}+\mathbf{b}_{+}^{m}=\mathbf{b}_{+}^{m}, \\
\lim _{R_{m} \rightarrow 0} \dot{F} & =\langle\tilde{\Phi}|H| \tilde{\Phi}\rangle,
\end{aligned}
$$

in agreement with (125).

Since the X-TDH working equations have singularities for some $\boldsymbol{\kappa}^{m}$ values (and thus some values of $R_{m}$ ), the possibility of the absence of global solutions has to be considered. A simple procedure (described in Sec. VI) can be implemented allowing the singularities to be avoided while propagating the modals.

The final working equations for the L-TDH and X-TDH methods are summarized in Table I.

\section{F. Calculation of the transformed integrals}

In order to construct the occupied-occupied and virtualoccupied blocks of the time-dependent one-mode integrals that are needed for expectation values and meanfield operators, we define the half-transformed matrix $\check{h}_{r^{m} s^{m}}^{m t}=\sum_{p^{m}} h_{r^{m} p^{m}}^{m t}\left[e^{\mathbf{K}^{m}}\right]_{p^{m} s^{m}}$. The time-dependent integrals are then obtained as $\tilde{h}_{r^{m} s^{m}}^{m t}=\sum_{p^{m}}\left[e^{-\mathbf{K}^{m}}\right]_{r^{m}} p^{m} \check{h}_{p^{m} s^{m}}^{m t}$. Using Eq. (91), we arrive at the expressions

$$
\begin{aligned}
\tilde{h}_{i^{m} i^{m}}^{m t}= & \cos \left(R_{m}\right) \check{h}_{i^{m} i^{m}}^{m t}+\operatorname{sinc}\left(R_{m}\right) I_{i^{m} i^{m}}^{m}, \\
\tilde{h}_{a^{m} i^{m}}^{m t}= & \check{h}_{a^{m} i^{m}}^{m t}-\operatorname{sinc}\left(R_{m}\right) \kappa_{a^{m} i^{m}}^{m} \check{h}_{i^{m} i^{m}}^{m t} \\
& -\frac{1}{2} \operatorname{sinc}^{2}\left(R_{m} / 2\right) \kappa_{a^{m} i^{m}}^{m} I_{i^{m} i^{m}}^{m},
\end{aligned}
$$

using the intermediates

$$
\begin{aligned}
& \check{h}_{i^{m} i^{m}}^{m t}=\cos \left(R_{m}\right) h_{i^{m} i^{m}}^{m t}+\operatorname{sinc}\left(R_{m}\right) \sum_{b^{m}} h_{i^{m} b^{m}}^{m t} \kappa_{b^{m} i^{m}}^{m}, \\
& \check{h}_{a^{m} i^{m}}^{m t}=\cos \left(R_{m}\right) h_{a^{m} i^{m}}^{m t}+\operatorname{sinc}\left(R_{m}\right) \sum_{b^{m}} h_{a^{m} b^{m}}^{m t} \kappa_{b^{m} i^{m}}^{m}, \\
& I_{i^{m} i^{m}}^{m}=\sum_{a^{m}} \kappa_{a^{m} i^{m}}^{m *} \check{h}_{a^{m} i^{m}}^{m t} .
\end{aligned}
$$

\section{G. Auto-correlation functions in X-TDH}

The time-dependent wave function can be used as a tool for calculating spectra by introducing the auto-correlation function $S(t),{ }^{1,8,37}$ which for $\mathrm{X}-\mathrm{TDH}$ takes the form [using Eq. (92)]

$$
S(t)=\langle\bar{\Phi}(0) \mid \bar{\Phi}(t)\rangle=e^{-i F(t)} \prod_{m} \cos \left(R_{m}\right) .
$$

TABLE I. Working equations for the L-TDH and X-TDH methods. Note that the L-TDH equations depend on the choice of the $g^{m}$ operator.

\begin{tabular}{cc}
\hline \hline L-TDH & X-TDH \\
\hline$i \dot{C}_{p^{m} i^{m}}^{m}=\sum_{q^{m}}\left[F_{p^{m} q^{m}}^{m \tilde{\mathbf{i}}}-\delta_{p^{m} q^{m}}\left(\tilde{F}_{i^{m} i^{m}}^{m} \tilde{\mathbf{i}}-\tilde{g}_{i^{m} i^{m}}^{m}\right)\right] C_{q^{m} i^{m}}^{m}$ & $\dot{\boldsymbol{\kappa}}_{+}^{m}=\frac{1}{\sin \left(R_{m}\right)}\left[\left(\frac{i y_{m}^{\mathfrak{J}}}{\cos \left(R_{m}\right)}+\operatorname{sinc}\left(R_{m}\right) y_{m}^{\mathfrak{R}}-y_{m}\right) \boldsymbol{\kappa}_{+}^{m}+R_{m} \mathbf{b}_{+}^{m}\right]$ \\
$\dot{F}=\sum_{t} c_{t} \prod_{m} \tilde{h}_{i^{m} i^{m}}^{m t}-\sum_{m=1}^{M} \tilde{g}_{i^{m} i^{m}}^{m}$ & $\dot{F}=\sum_{t} c_{t} \prod_{m} \tilde{h}_{i^{m} i^{m}}^{m t}+\sum_{m} \tan \left(R_{m}\right) y_{m}^{\mathfrak{J}}$ \\
\hline \hline
\end{tabular}


If the initial wave function is real and the Hamiltonian is symmetric, i.e., $H=H^{T}$, the following expression can be used for calculating the auto-correlation function at longer times:

$$
\begin{aligned}
S(2 t) & =\left\langle\bar{\Phi}^{*}(t) \mid \bar{\Phi}(t)\right\rangle \\
& =e^{-2 i F(t)} \prod_{m}\left[\cos ^{2}\left(R_{m}(t)\right)+\operatorname{sinc}^{2}\left(R_{m}(t)\right) \sum_{a^{m}}\left(\kappa_{a^{m} i^{m}}^{m}(t)\right)^{2}\right] .
\end{aligned}
$$

The absorption cross section at photon energy $E$ can then be obtained as the Fourier transform of $S(t), 8,37$

$$
\sigma(E) \sim E \int_{-\infty}^{\infty} S(t) e^{i\left(E_{i}+E\right) t} d t
$$

where $E_{i}$ is the energy of the initial wave packet.

\section{H. Computational scaling of X-TDH and L-TDH}

Comparing the X-TDH working equations [Eq. (132)] to the L-TDH counterpart (51) shows that in each derivative evaluation the $\mathrm{L}-\mathrm{TDH}$ method requires the construction of the full active-term mean-field matrix of size $N \times N$, where $N$ is the number of modals per mode (we here assume $N^{m}=$ $N \forall m$ ). X-TDH only needs the virtual-occupied block (one column vector of size $N-1$ ) which goes into the $\mathbf{b}_{+}^{m}$ vector. The X-TDH method thereby saves a factor $N$ in constructing the mean field which is the primary bottleneck for systems with many operator terms per mode (such as molecular PESs). When the operator contains few terms per mode or $N$ is very large, the bottleneck is instead matrix-vector products in the integral transformation scaling as $\mathcal{O}\left(N^{2}\right)$ for both methods.

\section{EXPONENTIAL PARAMETRIZATION OF EXACT AND VCI WAVE FUNCTIONS}

The exponential parameterization also covers the cases of an exact wave function and a truncated VCI wave function by a simple specialization. In this case, we remove the mode index and consider the shift operators $E_{P Q}$ as state-transfer operators, i.e., the operator

$$
E_{P Q}=|P\rangle\langle Q|
$$

transfers the system from state $|Q\rangle$ to $|P\rangle$. In addition to removing the $m$ index, we use capitalized letters to index the state space in a manner distinguishable from the one-mode analogs. Note that these state-transfer operators satisfy commutator relations corresponding to the commutator relations of the one-mode shift operator in Eq. (145),

$$
\left[E_{P Q}, E_{R S}\right]=\delta_{Q R} E_{P S}-\delta_{P S} E_{R Q}
$$

In this manner, the unitary transformations introduced in Sec. II B 3 are unitary transformations in the state space. Because the parameterization and commutator relations are equivalent, the same derivation applies, and the final result is essentially obtained by removing the $m$ index.

Thus, if we denote the reference state for the time evolution as $|I\rangle$ with an orthonormal set of basis states for its orthogonal complement indexed as $|A\rangle$, we can write states (VCI or exact or other) as

$$
|\tilde{\Phi}\rangle=\exp \left(\sum_{A}\left(K_{A}(t) E_{A I}-K_{A}^{*}(t) E_{I A}\right)\right)|I\rangle .
$$

Following the above derivation, the time evolution is given by

$$
\dot{\mathbf{K}}_{+}=\frac{1}{\sin (R)}\left[\left(\frac{i Y^{\mathfrak{I}}}{\cos (R)}+\operatorname{sinc}(R) Y^{\mathfrak{R}}-Y\right) \mathbf{K}_{+}+R \mathbf{B}_{+}\right]
$$

Here we have separated out the norm of the parameters as previously, i.e.,

$$
R=\left|\mathbf{K}_{+}\right| \text {. }
$$

The number $Y$ is obtained from

$$
Y=Y^{\mathfrak{R}}+i Y^{\mathfrak{I}}=\frac{1}{R} \mathbf{K}_{+}^{\dagger} \mathbf{B}_{+},
$$

in analogy to Eq. (128). The elements of the $\mathbf{B}_{+}$vector are obtained from the Hamiltonian matrix elements,

$$
\left[\mathbf{B}_{+}\right]_{A}=-i\langle\tilde{A}|H| \tilde{I}\rangle=-i \tilde{H}_{A I} .
$$

The tilde denotes that we are using the transformed-state basis. This can be related back to the integrals of the Hamiltonian in whatever primitive state basis is used. These relations follow the transformations in Subsection IV F, only the transformation is in state space instead of modal space. Altogether, the exact-state parameters can be evolved using the evolution of the unitary configuration-space parameters as given by Eq. (147). The right-hand side of this equation can be evaluated based on transformed Hamiltonian integrals. Inspecting the details, it is seen that the state can be propagated based on transformations of the Hamiltonian matrix followed by some dot products and vector additions. In this work, we focus on the efficiency gains of the exponential parameterization for TDH. Whether there are any gains of the formulation for exact or truncated time-dependent VCI calculations is a more complicated issue and will be investigated in the context of future work. We therefore do not present here the further details specializing the Hamiltonian matrix transformations, but all ingredients are available.

We note that the presented single-exponential parameterization presents an alternative to the standard linearly parameterized method for time evolution of any normalized state in an orthonormal basis. The type of parameterization is also directly relevant for a potential double-exponential parametrization of MCTDH, which is a topic for future research.

\section{IMPLEMENTATION SCHEME}

A wave-packet-propagation algorithm based on the TDH theory developed in Secs. II-IV is implemented in the Midas $\mathrm{Cpp}^{38}$ program package. The basic algorithms of the time-independent code can be reused, e.g., integral calculation and the active-terms algorithm for constructing the mean-field operator. ${ }^{6}$ The wave packet is represented in terms of a primitive time-independent basis which can be harmonic-oscillator functions, distributed Gaussians, ${ }^{39,40}$ or B-splines. ${ }^{41}$ Based on this primitive basis, an orthonormal basis is constructed in 
some way (e.g., using VSCF modals) and used in the TDH calculation.

The X-TDH and L-TDH implementations can be efficiently parallelized over both modes [using Message Passing Interface (MPI)] and operator terms (using OpenMP). This has been done in both the integral transformations and the derivative calculation where the loop over modes is parallelized using MPI and the loop over active terms in the mean-field construction is threaded using OpenMP.

For integrating the EOMs, we use our own implementation of the Dormand-Prince 8(5,3) (DOP853) explicit RungeKutta method ${ }^{42}$ with adaptive step-size control which also contains a high-order interpolant for dense output. The denseoutput feature is needed in order to obtain auto-correlation functions at equidistant time points which is required for using the Fast Fourier Transform (FFT) for calculating spectra. We have also implemented and tested the Dormand-Prince 5(4) (DOPRI5) method, ${ }^{42}$ but in our experience, the DOP853 method performs much better in terms of both speed and accuracy. It should also be noted that the step sizes used for integrating the L-TDH and X-TDH EOMs to a given accuracy are very similar in all test cases.

The singularities in the X-TDH EOMs are avoided by using the simple procedure described in the following.

1. Define a threshold $T_{\mathrm{R}} \in[0,1]$.

2. If at some point, $R_{m}>T_{\mathrm{R}} \times \frac{\pi}{2}$.

(a) As a first attempt, reduce the step size by a factor of two and retry the step.

(b) If this fails, transform the primitive integrals of mode $m$ using the last accepted step and reset $\boldsymbol{\kappa}^{m}$ to zero, i.e., $h_{r^{m} s^{m}}^{m t} \leftarrow \tilde{h}_{r^{m} s^{m}}^{m t}$ and $\boldsymbol{\kappa}^{m}=\mathbf{0}$, before retrying the rejected step.

The kappa vectors that have been used to transform the integrals need to be saved in order to calculate autocorrelation functions for later times. If at time $t$ the kappa vector has been reset at times $t_{1}^{*}<t_{2}^{*}<\ldots<t_{n}^{*}$, the auto-correlation functions at $t>t_{n}^{*}$ are calculated from the set of saved kappa vectors $\boldsymbol{\kappa}\left(t_{1}^{*}\right), \boldsymbol{\kappa}\left(t_{2}^{*}\right), \ldots, \boldsymbol{\kappa}\left(t_{n}^{*}\right)$ and the final kappa vector $\boldsymbol{\kappa}(t)$ by defining the total unitary-transformation matrix $\mathbf{U}^{m}(t)$,

$$
\begin{aligned}
\tilde{a}_{i^{m}}^{m \dagger}(t)= & \sum_{r^{m}} a_{r^{m}}^{m \dagger}\left(\sum_{s_{1}^{m}}\left[e^{\mathbf{K}^{m}\left(t_{1}^{*}\right)}\right]_{r^{m} s_{1}^{m}} \sum_{s_{2}^{m}}\left[e^{\mathbf{K}^{m}\left(t_{2}^{*}\right)}\right]_{s_{1}^{m} s_{2}^{m}} \ldots\right. \\
& \left.\times \sum_{s_{n+1}^{m}}\left[e^{\mathbf{K}^{m}(t)}\right]_{s_{n+1}^{m} i^{m}}\right)=\sum_{r^{m}} a_{r^{m}}^{m \dagger} U_{r^{m} i^{m}}^{m}(t) .
\end{aligned}
$$

The auto-correlation function is then calculated as

$$
S(t)=e^{-i F(t)} \prod_{m=1}^{M} U_{i^{m} i^{m}}^{m}(t),
$$

or under the conditions described above Eq. (142),

$$
S(2 t)=e^{-2 i F(t)} \prod_{m=1}^{M} \sum_{r^{m}}\left(U_{r^{m} i^{m}}^{m}(t)\right)^{2} .
$$

Note that Eqs. (152) and (153) reduce to Eqs. (141) and (142) when there are no reset kappa vectors.

\section{RESULTS}

\section{A. Computational details}

In the following, numerical results of the L-TDH and $\mathrm{X}-\mathrm{TDH}$ methods are presented. Calculations are performed on the set of polycyclic aromatic hydrocarbons (PAHs) of Ref. 6 which include up to two-mode couplings in the PESs and on high-dimensional Henon-Heiles systems.

As a primitive basis, we use $\mathrm{B}$-splines for all calculations. The initial wave packet is generated by performing a VSCF calculation on a second PES using the same primitive basis set. In this way, the vibrational wave function of one PES is placed on another potential on which the time propagation is performed. In order to obtain an orthonormal modal basis in which the reference state is contained, we transform from the primitive basis to the spectral basis of the VSCF calculation before starting the TDH propagation. This also allows us to truncate the basis based on the VSCF modal energies which may be done when the two PESs are similar and the wave packet does not change dramatically over time.

All results (except the ones in Subsection VII E) are obtained using the serial version of the TDH implementation.

\section{B. Dynamics of tetracene}

As a first test of the X-TDH method, we study the tetracene molecule (denoted as PAH4 in Ref. 6) with 84 vibrational modes and a PES including up to two-mode-coupling terms. The initial wave packet was generated by removing all couplings and $q^{n}$ terms with $n>2$ from the PES and performing VSCF on this potential. Propagation was performed for $t_{\text {end }}=10^{6}$ a.u. $\approx 24.2 \mathrm{ps}$ using the $N^{m}=48$ lowest VSCF modals as basis for each mode, and the resulting auto-correlation function is shown in Fig. 1 [note that we can calculate $S(t)$ at twice the time using Eq. (142)]. The auto-correlation function shows a significant structure. Because the PES is not harmonic, no complete revivals are observed in the studied time interval.

The spectrum obtained as the Fourier transform of $S(t)$ is shown in Fig. 2. As expected, the most intense peak corresponds to the VSCF ground-state energy $\left(E_{\mathrm{VSCF}}=2.397\right.$ $\times 10^{-1}$ a.u.) and a few small peaks are observed at higher energies. The intensities of the excited-state peaks are weak because the initial wave packet is quite similar to the VSCF state.

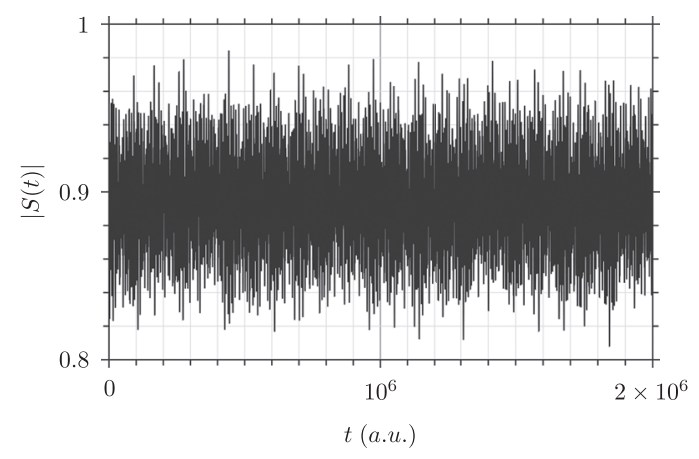

FIG. 1. Absolute value of the auto-correlation function of propagating tetracene from an initially Gaussian wave packet. 


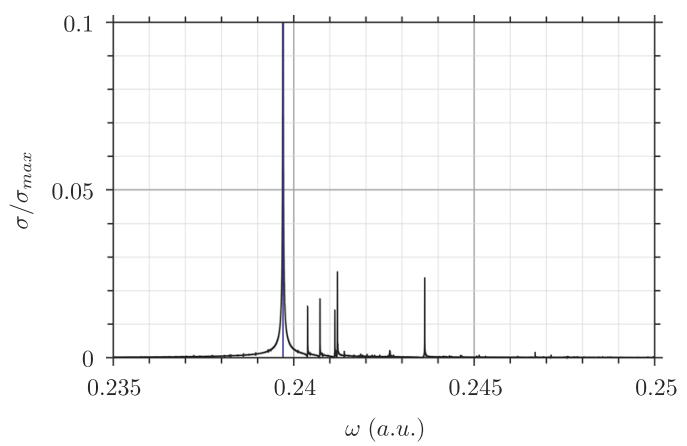

FIG. 2. Normalized spectrum of tetracene obtained from the Fourier transform of Fig. 1. The blue line in the middle of the most intense peak is the VSCF ground-state energy. Note that the top of the most intense peak (which is normalized to 1) is not shown in order to see the less intense features of the spectrum.

\section{Comparison of the L-TDH and X-TDH methods}

The L-TDH and X-TDH methods are two different parametrizations of the same wave function, and thus the results obtained from the two methods are the same. As discussed in Sec. IV $\mathrm{H}$, the X-TDH method only requires one column of the mean-field matrix, while the L-TDH method requires the whole matrix. This saves a factor of $N$ in the mean-field calculation and thus reduces the computational cost of propagating the TDH wave function significantly whenever the mean-field calculation is the primary bottleneck. This is the case when the PES contains many terms per mode which is likely to be the typical case and certainly true for the PAH examples considered here.

Figure 3 shows the average cost of a single propagation step for X-TDH and L-TDH calculations on PAHs. For L-TDH, $g^{m}=0$ is used (in our experience, changing the constraint operator has very little impact on the step sizes in the integrator). The initial wave packets are generated using the same procedure as in Sec. VII B and propagated to $t_{\text {end }}=$ $10^{3}$ a.u. Note that the number of propagation steps is approximately the same for both methods and thus the time per step reflects the total computation time. The data for each method have been fitted to a power function $f(M)=B M^{a}$, and in both cases, the scaling is close to the theoretical value $\mathcal{O}\left(M^{2}\right)$. The observed $M$ scaling is slightly lower for X-TDH because the cheaper mean-field calculation does not completely overshadow the cost of the integral transformations (which scale linearly with $M$ ) for the smaller molecules. It is clear from Fig. 3 that the X-TDH method is much faster than L-TDH. The gain of using X-TDH increases with $M$ because of the increasing number of mode couplings for larger molecules. For larger basis sets, the advantage of X-TDH is also larger but not twice as big for $N^{m}=64$ compared to $N^{m}=32$ because the cost of the integral transformations also increases.

\section{High-dimensional Henon-Heiles potentials}

In order to study the behavior for very large systems, we perform X-TDH calculations on the high-dimensional HenonHeiles potentials described in, e.g., Refs. 43 and 44,

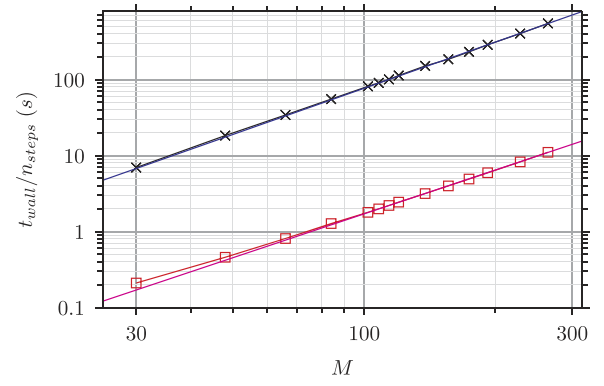

$\rightarrow \mathrm{L}-\mathrm{TDH}$

- $\mathrm{X}-\mathrm{TDH}$

$0.007012 M^{2.019992}$ $-0.000250 M^{1.917324}$

(a)

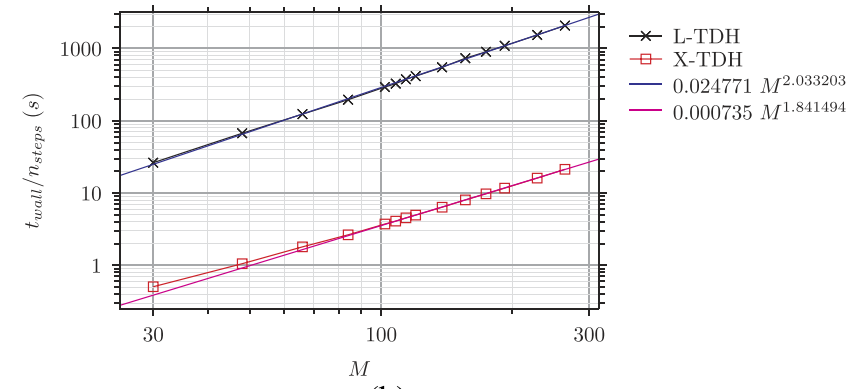

(b)

FIG. 3. $M$ scaling of the cost of a single step in propagating the L-TDH and $\mathrm{X}-\mathrm{TDH}$ EOMs for an array of PAHs using different numbers of modals. (a) $N^{m}=32$ and (b) $N^{m}=64$.

$$
H=-\frac{1}{2} \sum_{m=1}^{M} \frac{\partial^{2}}{\partial q_{m}^{2}}+\frac{1}{2} \sum_{m=1}^{M} q_{m}^{2}+\lambda \sum_{m=1}^{M-1}\left(q_{m}^{2} q_{m+1}-\frac{1}{3} q_{m+1}^{3}\right)
$$

with $\lambda=0.111803$. Since only nearest-neighbor couplings are included in the potential, the theoretical $M$ scaling of our TDH methods is linear. Note that this scaling is not trivial to obtain in practice for large systems. A series of calculations on systems with different numbers of dimensions have been performed where the initial wave packets were constructed as the groundstate wave function of a shifted harmonic oscillator with $V=\frac{1}{2} \sum_{m=1}^{M}\left(q_{m}-1\right)^{2}$. Note that because we have not implemented a complex absorbing potential (CAP) in MidasCpp, we had to use initial conditions different from the ones of Refs. 43 and 44 and thus the results are not easily comparable. Figure 4 shows the cost per step of the X-TDH method for calculations with up to 165000 dimensions, and it is clear that the scaling is very close to linear. The Henon-Heiles potential is a simple model system, but linear scaling of our TDH methods will also be observed for more complicated

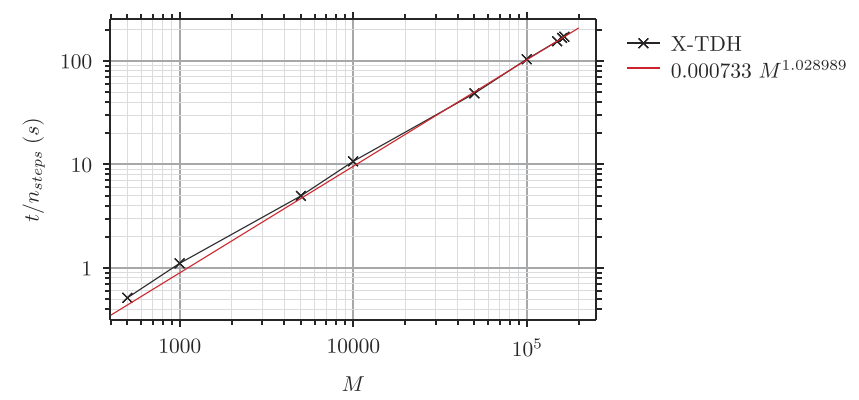

FIG. 4. $M$ scaling of the cost of a single step in propagating the X-TDHEOMs for high-dimensional Henon-Heiles potentials. 
10D Henon-Heiles

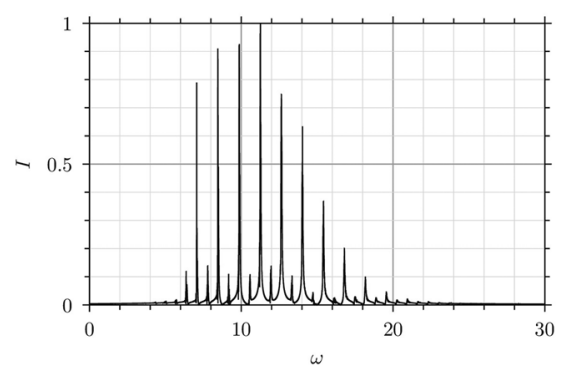

100D Henon-Heiles

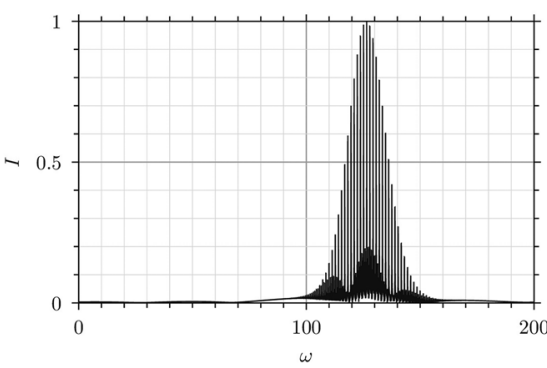

1000D Henon-Heiles

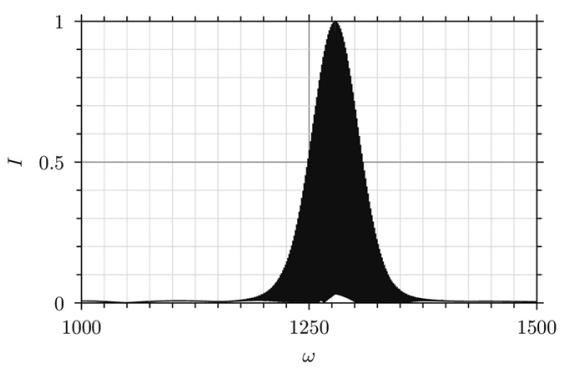

FIG. 5. Spectra of 10D, 100D, and 1000D Henon-Heiles potentials from propagation of a shifted Gaussian wave packet to $t_{\text {end }}=5 \times 10^{2}$ a.u. using X-TDH.

systems as long as the number of operator terms per mode is constant.

In Fig. 5, spectra of the 10D, 100D, and 1000D HenonHeiles systems are shown. As expected, the number of peaks (and thereby the number of states included in the initial wave packet) increases with the system size.

\section{E. Parallelization}

Figure 6 shows the parallel speedup in an X-TDH calculation on PAH8 (120 modes) with $N=48$ and $t_{\text {end }}=10^{3}$ a.u. using different numbers of threads for parallelizing over operator terms in the integral transformation and mean-field construction. The figure clearly shows that the wall time can be reduced significantly by using more CPUs. In Fig. 7, the parallel speedup is shown for using different numbers of MPI processes in the loops over modes in the integral transformation and derivative calculation. Also here, a significant parallel speedup is possible. Both data sets have been fitted to Amdahl's law,

$$
S(s)=\frac{1}{(1-p)+\frac{p}{s}},
$$

where $s$ is chosen to be the number of parallel processes in order to obtain an estimate for the parallel proportion of the execution time, $p$. In both cases, $p>90 \%$.

We can thus conclude that significant speedups can be obtained by parallelizing over either modes or operator terms. Which one is more favorable will depend on the calculation. For systems with many operator terms per mode (like the PAHs), approximately the same speedup can be obtained from both schemes. On the other hand, for high-dimensional systems with few coupling terms (like the Henon-Heiles systems), parallelization over modes will be most advantageous. The two levels of parallelism are also easily combined allowing for large-scale calculations on multiple computer nodes using both MPI and OpenMP. To give an absolute timing, on average one-time step (of average length $\sim 3$ a.u.) in the 264-mode $2.29 \times 10^{6}$-term PAH24 X-TDH computation takes $1.4 \mathrm{~s}$

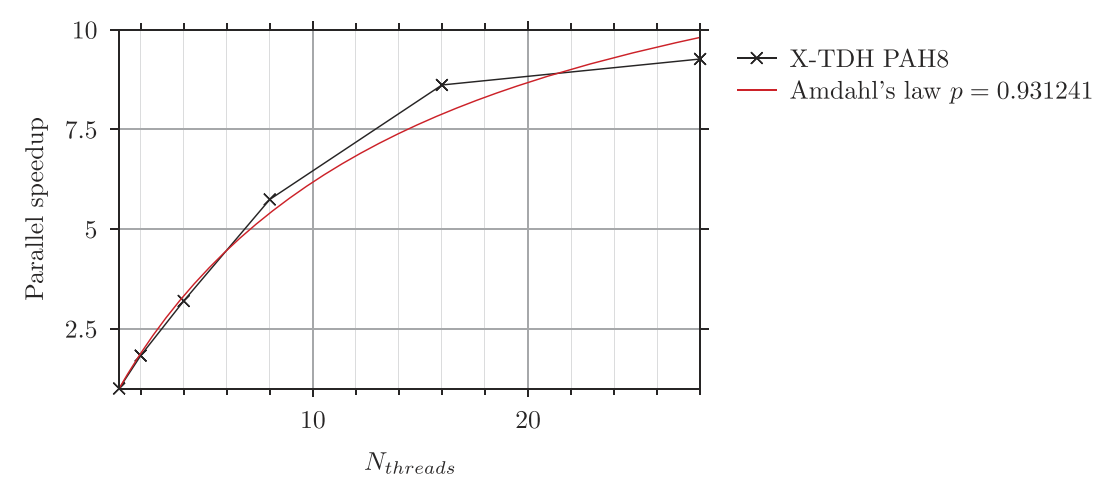

FIG. 6. Parallel speedup in integrating the X-TDH equations for PAH8 (120 modes) using different numbers of OpenMP threads in the integral transformations and mean-field construction. The data have been fitted to Amdahl's law.

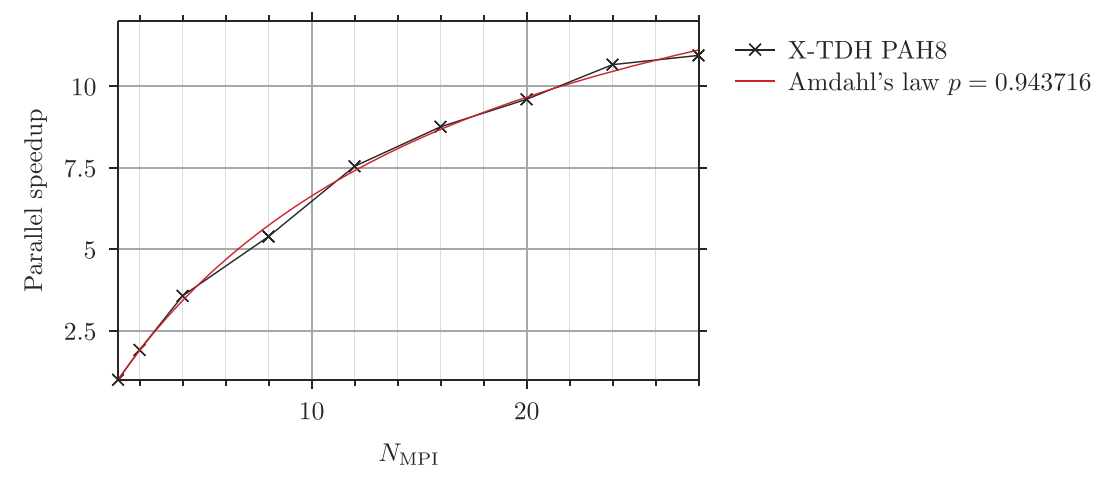

FIG. 7. Parallel speedup in integrating the X-TDH equations for PAH8 (120 modes) using different numbers of MPI processes in the integral transformations and derivative evaluation. The data have been fitted to Amdahl's law. 
with 12 MPI processes distributed over 3 nodes with the 7 OpenMP threads per MPI process. Note that the speedup from parallelizing over multiple nodes depends on the performance of the network.

\section{SUMMARY AND OUTLOOK}

We have introduced a new second-quantization formulation of TDH and derived equations of motion for both a linear (L-TDH) and an exponential (X-TDH) parametrization of the unitary modal transformations. The formulation also covers exact states as well as approximate VCI wave functions when the one-mode shift operators are replaced by state-transfer operators. The X-TDH method is significantly faster than L-TDH because it only requires one column of the mean-field matrix in order to propagate the EOMs. Numerical results which confirm the computational benefits of X-TDH have been presented for a set of PAHs with up to 264 modes and high-dimensional Henon-Heiles systems with more than $10^{5}$ modes. Thus, the X-TDH method paves the way for studying the quantum dynamics of very large systems.

The theoretical analysis of the exponential parametrization is an important step toward new formulations of correlated wave-function methods for solving the TDSE. For example, the exponential modal transformation can also be used in MCTDH and even an exponential parametrization of transformations in configuration space is possible using state-transfer operators. Thus, a new second-quantization formulation of MCTDH which benefits from the active-terms algorithm in the mean-field calculation and employs linear and/or exponential parametrizations of the modal and configuration-space transformations is an interesting topic for future research. In order to avoid the exponential scaling of MCTDH with respect to the system size, we are currently developing a time-dependent version of VCC theory. Here, the present TDH work becomes important when time-dependent modals are introduced in the VCC wave function. Thus, the new TDH formulation is a relevant method in its own right for studying large quantum systems, and the mathematical analysis of the exponential modal transformations paves the way for developing new accurate methods for solving the time-dependent Schrödinger equation.

\section{ACKNOWLEDGMENTS}

O.C. acknowledges support from the Danish Council for Independent Research through a Sapere Aude III grant (No. DFF-4002-00015), the Lundbeck Foundation, and the Danish e-infrastructure Cooperation (DeiC). O.C. acknowledges discussions with Henrik Larsson.

\footnotetext{
${ }^{1} \mathrm{D}$. Tannor, Introduction to Quantum Mechanics: A Time-Dependent Perspective (University Science Books, Sausalito, California, USA, 2005).

${ }^{2}$ N. Makri, Annu. Rev. Phys. Chem. 50(1), 167 (1999).

${ }^{3}$ J. M. Bowman, J. Chem. Phys. 68(2), 608 (1978).

${ }^{4}$ R. B. Gerber and M. A. Ratner, Adv. Chem. Phys. 70, 97 (1988).
}

${ }^{5}$ J. M. Bowman, Acc. Chem. Res. 19(7), 202 (1986).

${ }^{6}$ M. Hansen, M. Sparta, P. Seidler, D. Toffoli, and O. Christiansen, J. Chem. Theory Comput. 6(1), 235 (2010).

${ }^{7}$ O. Christiansen, J. Chem. Phys. 120, 2140 (2004).

${ }^{8}$ M. Beck, A. Jackle, G. Worth, and H. Meyer, Phys. Rep. 324, 1 (2000).

${ }^{9}$ H.-D. Meyer, U. Manthe, and L. S. Cederbaum, Chem. Phys. Lett. 165(1), 73 (1990).

${ }^{10}$ H. Wang and M. Thoss, J. Chem. Phys. 119, 1289 (2003).

${ }^{11}$ U. Manthe, J. Chem. Phys. 128, 164116 (2008).

${ }^{12}$ H. Wang, J. Phys. Chem. A 119(29), 7951 (2015).

${ }^{13}$ I. Burghardt, M. Nest, and G. A. Worth, J. Chem. Phys. 119(11), 5364 (2003).

${ }^{14}$ M. Bonfanti, G. F. Tantardini, K. H. Hughes, R. Martinazzo, and I. Burghardt, J. Phys. Chem. A 116(46), 11406 (2012).

${ }^{15}$ I. Burghardt, H. Meyer, and L. Cederbaum, J. Chem. Phys. 111, 2927 (1999).

${ }^{16}$ G. Richings, I. Polyak, K. Spinlove, G. Worth, I. Burghardt, and B. Lasorne, Int. Rev. Phys. Chem. 34(2), 269 (2015).

${ }^{17}$ J. A. Green, A. Grigolo, M. Ronto, and D. V. Shalashilin, J. Chem. Phys. 144(2), 024111 (2016).

${ }^{18}$ B. F. E. Curchod and T. J. Martínez, Chem. Rev. 118(7), 3305 (2018).

${ }^{19}$ O. Christiansen, J. Chem. Phys. 120(5), 2149 (2004).

${ }^{20}$ P. Seidler and O. Christiansen, J. Chem. Phys. 131(23), 234109 (2009).

${ }^{21}$ B. Thomsen, M. B. Hansen, P. Seidler, and O. Christiansen, J. Chem. Phys. 136(12), 124101 (2012).

${ }^{22}$ I. H. Godtliebsen and O. Christiansen, J. Chem. Phys. 143(13), 134108 (2015).

${ }^{23}$ N. K. Madsen, I. H. Godtliebsen, S. A. Losilla, and O. Christiansen, J. Chem. Phys. 148(2), 024103 (2018).

${ }^{24}$ H.-D. Meyer, F. Gatti, and G. A. Worth, Multidimensional Quantum Dynamics: MCTDH Theory and Applications (Wiley-VCH, Weinheim, 2009).

${ }^{25}$ F. Gatti, B. Lasorne, H.-D. Meyer, and A. Nauts, Applications of Quantum Dynamics in Chemistry, Volume 98 of Lecture Notes in Chemistry (Springer, 2017).

${ }^{26} \mathrm{~A}$. Zoccante, "Time-dependent and time-independent vibrational coupled cluster theory," Ph.D. thesis (Aarhus University, 2012).

${ }^{27}$ T. Helgaker, P. Jørgensen, and J. Olsen, Molecular Electronic Structure Theory (Wiley, Chichester, 2000).

${ }^{28}$ P. A. M. Dirac, The Principles of Quantum Mechanics, The International Series of Monographs on Physics, edited by R. H. Fowler and P. Kapitzer (Clarendon Press, Oxford, 1930).

${ }^{29}$ A. D. McLachlan and M. A. Ball, Rev. Mod. Phys. 36(3), 844 (1964).

${ }^{30}$ R. Gerber, V. Buch, and M. Ratner, Chem. Phys. Lett. 91, 173 (1982).

${ }^{31} \mathrm{P}$. Kramer and M. Saraceno, Geometry of the Time-Dependent Variational Principle in Quantum Mechanics (Springer Verlag, Berlin, 1981).

${ }^{32}$ P. Löwdin and P. Mukherjee, Chem. Phys. Lett. 14, 1 (1972).

${ }^{33}$ O. Christiansen, P. Jørgensen, and C. Hättig, Int. J. Quantum Chem. 68, 1 (1998).

${ }^{34}$ R. M. Wilcox, J. Math. Phys. 8(4), 962 (1967).

${ }^{35}$ R. Penrose, "A general inverse for matrices," Math. Proc. Cambridge Philos. Soc. 51, 406-413 (1955).

${ }^{36}$ O. Christiansen, J. Chem. Phys. 122(19), 194105 (2005).

${ }^{37}$ R. Schinke, Photodissociation Dynamics: Spectroscopy and Fragmentation of Small Polyatomic Molecules, Cambridge Monographs on Atomic, Molecular and Chemical Physics (Cambridge University Press, 1993).

${ }^{38}$ O. Christiansen, I. H. Godtliebsen, E. M. Gras, W. Gyốrffy, M. B. Hansen, M. B. Hansen, J. Kongsted, E. L. Klinting, C. König, S. A. Losilla, D. Madsen, N. K. Madsen, K. Monrad, P. Seidler, K. Sneskov, M. Sparta, B. Thomsen, D. Toffoli, and A. Zoccante, MidasCpp (Molecular Interactions, Dynamics and Simulation Chemistry Program Package in C++) (University of Aarhus, 2018), www.chem.au.dk/midas.

${ }^{39}$ I. P. Hamilton and J. C. Light, J. Chem. Phys. 84(1), 306 (1986).

${ }^{40}$ B. Poirier and J. C. Light, J. Chem. Phys. 113(1), 211 (2000).

${ }^{41}$ D. Toffoli, M. Sparta, and O. Christiansen, Mol. Phys. 109(5), 673 (2011).

${ }^{42}$ E. Hairer, G. Wanner, and S. P. Nørsett, Solving Ordinary Differential Equations. I. Nonstiff Problems (Springer, 1993).

${ }^{43}$ M. Nest and H.-D. Meyer, J. Chem. Phys. 117(23), 10499 (2002).

${ }^{44}$ O. Vendrell and H.-D. Meyer, J. Chem. Phys. 134(4), 044135 (2011). 